\title{
A deep-learning estimate of the decadal trends in the Southern Ocean carbon storage
}

By Varvara E. Zemskova (barbara.zemskova@utoronto.ca),

Tai-Long He (tailong.he@mail.utoronto.ca),

Zirui Wan,

and

Nicolas Grisouard (nicolas.grisouard@utoronto.ca)

University of Toronto, Department of Physics, 60 St. George Street, Toronto ON M5S 1A7, Canada

This paper is a non-peer reviewed preprint submitted to EarthArXiv.

It was submitted to Nature Communications on 12 April, 2021 and is currently undergoing peer review.

Please feel free to reach out to us if you have any questions or comments regarding the paper. 


$$
{ }_{6} \text { anth }
$$

$$
8 \text { ocea }
$$

\title{
17 Introduction
}

\section{A deep-learning estimate of the decadal trends in the Southern Ocean carbon storage}

\author{
Varvara E. Zemskova*1, Tai-Long He ${ }^{\dagger 1}$, Zirui Wan ${ }^{1}$, and Nicolas Grisouard ${ }^{1}$ \\ ${ }^{1}$ Department of Physics, University of Toronto, Toronto, ON, Canada
}

Uptake of atmospheric carbon by the ocean, especially at high latitudes, plays an important role in offsetting anthropogenic emissions ${ }^{1,2}$. At the surface of the Southern Ocean south of $30^{\circ} \mathrm{S}$, the ocean carbon uptake, which had been weakening in $1990 \mathrm{~s}$, strengthened in the $2000 \mathrm{~s}^{3,4}$. However, sparseness of in-situ measurements in the ocean interior make it difficult to compute changes in carbon storage below the surface ${ }^{5,6}$. Here we develop a machine-learning model, which estimates concentrations of dissolved inorganic carbon (DIC) in the Southern Ocean up to $4 \mathrm{~km}$ depth only using data available at the ocean surface. We applied this model to calculate rends in DIC concentrations over the past three decades and found that DIC decreased in the upper $1 \mathbf{~ k m}$ and increased in deeper layers over this period. However, the particular circulation dynamics that drove these hanges may have differed across zonal sectors of the Southern Ocean. While the near-surface decrease in DIC concentrations would enhance atmospheric $\mathrm{CO}_{2}$ uptake continuing the previously-found trends, weakened connectivity between surface and deep layers and build-up of DIC in deep waters could reduce the ocean's carbon storage potential.

Atmospheric $\mathrm{CO}_{2}$ concentrations have been rising since the pre-industrial era, in large part due to burning of the fossil fuels and land-use changes, such as deforestation and urbanization ${ }^{7,8}$. Global carbon budget models estimate that oceans absorb about $25 \%$ of anthropogenic carbon emissions ${ }^{1}$. Polar regions play a particularly important role in carbon uptake, i.e., the transfer of $\mathrm{CO}_{2}$ from air into the ocean. Indeed, carbon uptake increases with decreasing temperature and increasing wind speed, which enhances mixing at the surface ${ }^{2}$. Consequently, it is estimated that the Southern Ocean is responsible for approximately $40 \%$ of the anthropogenic carbon sink ${ }^{9}$, where persistent zonal winds re strong and temperatures are relatively cold.

There has been concern regarding a declining trend in the Southern Ocean carbon uptake from the 1980s into early $2000 \mathrm{~s}^{10,11}$. However, recent multidecadal analysis of surface ocean $\mathrm{CO}_{2}$ measurements found a reversed trend, i.e. that the ocean carbon uptake has been increasing in the 2000s, attributed to changes in ocean circulation, which are primarily due to non-trivial shifts in wind forcing ${ }^{3}$. However, carbon needs to be exported from the surface down into the ocean interior, where it cannot further exchange with the atmosphere ${ }^{12}$. The changes in this export are important not only for the climate but also marine chemistry. An increase in dissolved carbon has led to ocean acidification that subsequently affects marine organisms ${ }^{13}$. However, trends in carbon concentrations in the ocean interior are still poorly understood, primarily for two reasons. First, it is difficult to model biogeochemical cycles in ocean models ${ }^{14}$ and second, ocean measurements are spatially and temporally sparse ${ }^{5,6}$.

To address this sparseness of observations, we developed a neural network mode ${ }^{15}$ that predicts concentrations of dissolved inorganic carbon (DIC) in the upper $4 \mathrm{~km}$ in the ocean using surface and near-surface variables: sea surface temperature, flow velocity at the surface, sea surface height, near-surface wind velocity, surface $\mathrm{CO}_{2}$ partial pressure $\left(p \mathrm{CO}_{2}\right)$. All of the input parameters are readily available via satellite measurements, with the exception of $p \mathrm{CO}_{2}$, which has been previously estimated by another neural network ${ }^{16}$ trained and tested with observational data from Surface Ocean $\mathrm{CO}_{2}$ Atlas (SOCAT).

We train our model in two phases (see Methods): first us the Biogeochemical Southern Ocean State Estimate (BSOSE), which is a data assimilating ocean circulation model $^{14}$. It is available at a high spatial and temporal resolution of $1 / 3^{\circ}$ and 3 -day resolution, respectively, and therefore provides a large volume of data for the initial training. In the second phase, we use DIC measurements from Global Ocean Data Analysis Project version 2 (GLODAPv2) shipboard measurements $\mathrm{s}^{17,18}$ and Southern Ocean Carbon and Climate Observations and Modeling (SOCCOM) biogeochemical Argo floats ${ }^{19}$. These measurements are used to correct any biases originating from the B-SOSE model used in the first phase. Similar to previous works on modeling $p \mathrm{CO}_{2}{ }^{20}$, we find that the model relative error is reduced when using a combination of shipboard and float measurements in the training set.

\section{Observed decadal trends}

Using this neural network model, we computed the distribution of five day-averaged DIC concentrations over the $993-2019$ period south of $30^{\circ} \mathrm{S}$. The depth- and zonally-averaged DIC concentrations (separated into three ocean basins: Atlantic, Pacific, and Indian) are shown in Extended Data Fig. 1 and averaged over three periods (1993-1999, 2000-2009, 2010-2019). Near the surface, DIC concentrations increase polewards with latitude and largely follow the the neutral density surfaces in the interior, consistent with previous estimate ${ }^{21}$. The Pacific and Indian basins, which have older, bottom-sourced waters ${ }^{22}$ have higher DIC concentrations compared with the Atlantic basin, whose deep waters are ventilated more frequently ${ }^{22}$.

*barbara.zemskova@utoronto.ca

†tailong.he@mail.utoronto.ca 
Overall between 1993 and 2019, DIC concentrations have been decreasing near the surface (Figs. 1a-c) and increasing in the deep ocean (Figs. 1d-f). The decreasing surface DIC trend, which subsequently lowers $p \mathrm{CO}_{2}$ at the ocean surface, is consistent with the previously found strengthening of the Southern Ocean carbon sink in the $2000 \mathrm{~s}^{3}$. Our analysis shows that this trend continued into the 2010s. However, the changes in DIC concentrations are not zonally uniform, suggesting that distinct mechanisms may exist in different ocean basins (cf. Extended Data Fig. 2).

In the 1990s, DIC mostly increased in the upper $1 \mathrm{~km}$ over the Pacific within the Antarctic Circumpolar region $\left(50-60^{\circ} \mathrm{S}\right.$; Figs. 1a, 2d). The predominantly positive phase of the Southern Annular Mode since the $1980 \mathrm{~s}^{23,24}$ has been associated with the intensification and poleward shift in the Westerlies, the zonally persistent eastward winds at these latitudes. These stronger winds result in flow divergence near the surface and intensify upwelling of DIC-rich waters from the abyss ${ }^{25}$. Consistent with the signature of stronger upwelling, there is a decrease in DIC in deeper waters (Fig. 1d).

While there is also an increasing DIC trend in the South Atlantic and South Indian Oceans in the 1990s along the upwelling isosurfaces at these latitudes (Figs. 1a, 2a,g), the rates are lower than in the South Pacific. The zonal differences could be attributed to the zonal asymmetry in the atmospheric forcing ${ }^{26}$ that has resulted in greater intensification of the Westerlies over the Pacific than the Atlantic or Indian sectors ${ }^{3,27}$. The overall increase in DIC is further consistent with the increase in sea surface $p \mathrm{CO}_{2}$ and increased outgassing or decreased uptake of atmospheric carbon by the Southern Ocean in response to the positive Southern Annular Mode 10,11,28. Notably, the strong near-surface negative trend in the Western Indian sector around $40-50^{\circ} \mathrm{S}$ could be because of the increased stratification due to warming in this region over the previous several decades ${ }^{29}$.

In addition to an increase in upwelling, stronger Westerlies in the Southern Hemisphere also lead to an increase in northward Ekman transport ${ }^{3}$, which at the surface brings sea ice and colder and fresher water from the Antarctic coast. Indeed, decreasing sea surface temperatures ${ }^{30,31,32}$ and increasing freshwater fluxes due to northward sea-ice transport and increased precipitation ${ }^{33}$ have been observed over the South Pacific sector starting in the 2000s. To understand the circulation in the Pacific and its role in transport of DIC, we consider effects on water-mass classes of specific neutral density $\left(\gamma_{n}\right)$ ranges: Circumpolar Deep Water $\left(\mathrm{CDW}, \gamma_{n}=27.5-28 \mathrm{~kg} / \mathrm{m}^{3}\right.$ ), Antarctic Intermediate Water (AAIW, $\gamma_{n}=27.0-27.5 \mathrm{~kg} / \mathrm{m}^{3}$ ), and Subantarctic Mode Water (SAMW, $\left.\gamma_{n}=26.6-27.0 \mathrm{~kg} / \mathrm{m}^{3}\right)^{34}$. CDW comprises of old, dense waters that upwell to the surface south of $55^{\circ} \mathrm{S}$; in South Atlantic, this water-mass is North Atlantic Deep Water (NADW). AAIW comprises of cold and fresh waters that travel northward from the upwelling zone and eventually sink to about $1 \mathrm{~km}$ depth, and SAMW of upwelled waters that continue to travel equatorward at the surface before sinking $^{22}$ (cf. isocontours in Fig. 2).

A water-mass can gain buoyancy (become lighter) due to ice melt or lose buoyancy (become denser) due to brine rejection at the surface. In mid- to late-2000s, an increase in melting of advected ice contributed to buoyancy gain of SAMW within the upper $700 \mathrm{~m}^{34}$, which was made even lighter by surface heating north of $40^{\circ} \mathrm{S}^{30}$. Increased freshwater flux from ice melt also has made AAIW lighter, counteracting the buoyancy loss due to cooling at the surface $^{34,35}$. In contrast, salt fluxes due to brine rejection led to buoyancy loss of CDW, but with large zonal differences. In the Atlantic sector (Weddell Sea), destruction of water-masses in the $27.6-27.8 \mathrm{~kg} / \mathrm{m}^{3}$ neutral density range near the surface ${ }^{34}$ required that water in this density range was upwelled from the interior. However, in the Pacific sector (Ross Sea), positive formation rates of this density range near the surface ${ }^{34}$ weakened the upwelling.

These water-mass transformations can help explain the DIC trends in the Pacific that we find in the 2000s and 2010s. Weakening of CDW upwelling south of $60^{\circ} \mathrm{S}$ resulted in decreased delivery of old DIC-rich waters to the surface, and hence a weaker increasing trend in DIC near the surface in 2000s (Figs. 1b, 2e). In the 2010s, the near-surface DIC trends further decreased and became negative (Figs. 1c, 2f), while DIC built up (increasing trend) below $1 \mathrm{~km}$ depth at the latitudes of CDW upwelling (Figs. 1f, 2f). The decreasing DIC trends follow the AAIW and SAMW density isosurfaces northward, further pointing to weakened upwelling being responsible, as the upwelled CDW comprises a large portion of AAIW and SAMW. Climatologically, these findings are important because a decrease in near-surface DIC concentrations can enhance the uptake of atmospheric carbon by the ocean. However, recent satellite measurements ${ }^{32}$ found increasing sea surface temperatures over much of the Pacific sector in the 2010s, which may reverse the DIC trends found in this study. While the response of the deeper overturning and upwelling will be delayed, the buoyancy gain of AAIW at surface could stabilize the water column and decrease the export of the DIC from the surface below the mixed layer depth $\left(\sim 200-600 \mathrm{~m}^{36}\right)$ to be sequestered.

Unlike the Pacific, most of the Atlantic and Indian sectors of the Southern Ocean, especially between $30-60^{\circ} \mathrm{S}$ have been warming and storing heat in the upper $2 \mathrm{~km}$ over the past few decades ${ }^{37,38}$. The larger heat uptake over the Southern Ocean compared with the northern temperate and high-latitudes is partially because of the reinforcement of greenhouse gas-induced heating by ozone-hole forcing ${ }^{39}$ and low levels of aerosols, which could have a cooling effect $^{38}$, in the Southern Hemisphere. Warming of the upper ocean stabilizes the water column, weakening the effects of the wind-driven upwelling around $50-55^{\circ} \mathrm{S}$. In the Atlantic sector, these changes are reflected in a decrease of a positive trend in DIC concentrations along the upwelling density isosurfaces in 2000s compared with 1990s (Figs. 2ab). In the upper $1 \mathrm{~km}$, trends are predominantly negative between $45-60^{\circ} \mathrm{S}$ in the $2010 \mathrm{~s}$, reflecting the trends of the SAMW/AAIW zonally advected from the Pacific sector (Figs. 1c, 2c). In the Indian sector, we find similar negative trends south of $50^{\circ} \mathrm{S}$, but positive trends near the surface to the north (Figs. 1c, 2h,i). The regions of near-surface positive trends correspond to areas, where strong SAMW and AAIW formation rates ${ }^{40,41}$ are enhanced by salinity fluxes ${ }^{42}$ and can help export DIC into the interior (Fig. 2b,c).

Furthermore, Atlantic Meridional Overturning Circulation (AMOC) has been weakening since the $1990 \mathrm{~s}^{43,44,45}$. AMOC transports dense water sinking in the North Atlantic to the upwelling region in the South Atlantic. The slowdown of AMOC has been attributed to increased uptake of heat by the North Atlantic in response to rising atmospheric greenhouses gas levels ${ }^{43}$ and weakening of North Atlantic Oscillation since the early $1990 \mathrm{~s}^{38,46}$. As a result, meridional transport has weakened and due to buoyancy gain, surface waters in the North Atlantic have been sinking to shallower depths, where DIC content is lower. These changes in the circulation dynamics, which diminish the connectivity between the deep and surface layers, are consistent with our results: progressively decreasing trends along the upwelling density isosurfaces from 1990s to 2010s, especially near the surface, and an increase in DIC concentrations in the interior below $1 \mathrm{~km}$ depth in the 2010s. Since mid-2010s, increased AMOC transport has been recorded in the subtropics in the Northern Hemisphere ${ }^{47,46}$. However, because of the long temporal scales in ocean circulation, there will be a 


\section{Discussion}

Our results show an overall decreasing trend in DIC concentrations in the upper part (top $1 \mathrm{~km}$ ) of the Southern Ocean over the period from 1993 to 2019. This trend is congruent with the previous findings of decreasing $\mathrm{CO}_{2}$ uptake in this region in the $1990 \mathrm{~s}$ and increasing uptake in the $2000 \mathrm{~s}^{3,4}$, and indicate the continuation of the increasing uptake potential at the ocean surface into the 2010s. These findings, however, are contrary to an overall increase in DIC concentrations found recently ${ }^{6}$, which computed the decadal changes by comparing the spatially-interpolated data only from biogeochemical floats over the $2014-2019$ period with shipboard measurements prior to 2005 . While floats can augment shipboard data, especially because of superior wintertime coverage, it has been found that models using only data from floats can underestimate the Southern Ocean carbon uptake by threefold compared with models only using shipboard data and by twofold compared with models using a combination of float and shipboard data ${ }^{20}$. Thus, using only float data in the 2010 s could overestimate the DIC concentrations over this time period (subsequently underestimating the carbon uptake at the surface). It would then yield positive long-term DIC trends, contrary to the negative trends found by our model, which integrates data from both shipboard measurements and Argo floats.

Our results demonstrate similar effects of weakening upwelling and connectivity between the deep and surface waters in different sectors of the Southern Ocean. Although these trends are in line with the expected changes in ocean circulation, what drives these changes varies zonally. The difference in the underlying mechanisms implies that responses to future changes in the circulation dynamics may also not be zonally uniform. In the current model, we are unable to separate changes in DIC concentrations due to uptake of anthropogenic carbon and due to natural variability in the ocean circulation; it may be pertinent to include methods from previous studies ${ }^{5,6}$ into future analysis. Nevertheless, the decrease in DIC concentrations near the surface that we observe allows for increased uptake of carbon from the atmosphere. However, continued monitoring efforts are necessary to assess the long-term impacts of DIC accumulation on storage of anthropogenic $\mathrm{CO}_{2}$ in the deep ocean. These changes are important not only from a climatological point of view, but also for the management of marine ecosystems, which are sensitive to acidification ${ }^{48}$. The model presented here can serve as a useful tool for such future studies as it is able to estimate DIC concentrations in the ocean interior up to $4 \mathrm{~km}$ depth from new satellite measurements as they become available.

\section{References}

[1] Corinne Le Quéré et al. “Global carbon budget 2018”. In: Earth System Science Data 10.4 (2018), pp. 21412194.

[2] Taro Takahashi et al. "Climatological mean and decadal change in surface ocean pCO2, and net sea-air CO2 flux over the global oceans”. In: Deep Sea Research Part II: Topical Studies in Oceanography 56.8-10 (2009), pp. 554-577.

[3] Peter Landschützer et al. "The reinvigoration of the Southern Ocean carbon sink”. In: Science 349.6253 (2015), pp. 1221-1224.

[4] Tim DeVries, Mark Holzer, and Francois Primeau. "Recent increase in oceanic carbon uptake driven by weaker upper-ocean overturning”. In: Nature 542.7640 (2017), pp. 215-218.

[5] Nicolas Gruber et al. "The oceanic sink for anthropogenic CO2 from 1994 to 2007”. In: Science 363.6432 (2019), pp. 1193-1199.

[6] Ben Bronselaer et al. "Importance of wind and meltwater for observed chemical and physical changes in the Southern Ocean”. In: Nature Geoscience 13.1 (2020), pp. 35-42.

[7] Philippe Ciais et al. "Carbon and other biogeochemical cycles". In: Climate change 2013: the physical science basis. Contribution of Working Group I to the Fifth Assessment Report of the Intergovernmental Panel on Climate Change. Cambridge University Press, 2014, pp. 465-570.

[8] Lucy R Hutyra et al. "Urbanization and the carbon cycle: Current capabilities and research outlook from the natural sciences perspective”. In: Earth's Future 2.10 (2014), pp. 473-495.

[9] Tim DeVries. "The oceanic anthropogenic CO2 sink: Storage, air-sea fluxes, and transports over the industrial era”. In: Global Biogeochemical Cycles 28.7 (2014), pp. 631-647.

[10] Corinne Le Quéré et al. "Response to comments on" Saturation of the Southern Ocean CO2 sink due to recent climate change"", In: science 319.5863 (2008), pp. 570-570.

[11] Nicole S Lovenduski, Nicolas Gruber, and Scott C Doney. "Toward a mechanistic understanding of the decadal trends in the Southern Ocean carbon sink". In: Global Biogeochemical Cycles 22.3 (2008).

[12] Dan Jones and Takamitsu Ito. "Gaussian mixture modeling describes the geography of the surface ocean carbon budget." In: Proceedings of the 9th International Workshop on Climate Informatics (2019).

[13] Katherina Petrou et al. "Acidification diminishes diatom silica production in the Southern Ocean". In: Nature Climate Change 9.10 (2019), pp. 781-786.

[14] Ariane Verdy and Matthew R Mazloff. "A data assimilating model for estimating S outhern O cean biogeochemistry". In: Journal of Geophysical Research: Oceans 122.9 (2017), pp. 6968-6988.

[15] Tai-Long He et al. "Recurrent U-net: Deep learning to predict daily summertime ozone in the United States". In: arXiv preprint arXiv:1908.05841 (2019).

[16] Peter Landschützer et al. "A neural network-based estimate of the seasonal to inter-annual variability of the Atlantic Ocean carbon sink”. In: Biogeosciences 10.11 (2013), pp. 7793-7815.

[17] Are Olsen et al. "GLODAPv2. 2020-the second update of GLODAPv2". In: Earth System Science Data Discussions (2020), pp. 1-41. 
[18] Robert M Key et al. “Global ocean data analysis project, version 2 (GLODAPv2)”. In: Ornl/Cdiac-162, Ndp-093 (2015).

[19] Kenneth S. Johnson et al. "Southern Ocean Carbon and Climate Observations and Modeling (SOCCOM) Float Data Archive". In: (2019). URL: https ://doi .org/10.6075/J01G0JKT.

[20] Seth M Bushinsky et al. "Reassessing Southern Ocean air-sea CO2 flux estimates with the addition of biogeochemical float observations”. In: Global biogeochemical cycles 33.11 (2019), pp. 1370-1388.

[21] Yingxu Wu et al. "What drives the latitudinal gradient in open-ocean surface dissolved inorganic carbon concentration?" In: Biogeosciences 16.13 (2019), pp. 2661-2681.

[22] Lynne D Talley. "Closure of the global overturning circulation through the Indian, Pacific, and Southern Oceans: Schematics and transports”. In: Oceanography 26.1 (2013), pp. 80-97.

[23] Gareth J Marshall et al. "Causes of exceptional atmospheric circulation changes in the Southern Hemisphere". In: Geophysical Research Letters 31.14 (2004).

[24] Alexander Sen Gupta and Matthew H England. "Coupled ocean-atmosphere-ice response to variations in the southern annular mode". In: Journal of Climate 19.18 (2006), pp. 4457-4486.

[25] Darryn W Waugh et al. "Recent changes in the ventilation of the southern oceans". In: Science 339.6119 (2013), pp. 568-570

[26] Marilyn N Raphael et al. "The Amundsen Sea low: Variability, change, and impact on Antarctic climate". In: Bulletin of the American Meteorological Society 97.1 (2016), pp. 111-121.

[27] Andrea F Carril and Antonio Navarra. "Low-frequency variability of the Antarctic Circumpolar Wave". In: Geophysical Research Letters 28.24 (2001), pp. 4623-4626.

[28] Carolina O Dufour et al. "Eddy compensation and controls of the enhanced sea-to-air CO2 flux during positive phases of the Southern Annular Mode”. In: Global Biogeochemical Cycles 27.3 (2013), pp. 950-961.

[29] Friedrich A Schott, Shang-Ping Xie, and Julian P McCreary Jr. "Indian Ocean circulation and climate variability". In: Reviews of Geophysics 47.1 (2009).

[30] Dean Roemmich et al. "Unabated planetary warming and its ocean structure since 2006". In: Nature climate change 5.3 (2015), pp. 240-245.

[31] Yavor Kostov et al. "Contributions of greenhouse gas forcing and the Southern Annular Mode to historical Southern Ocean surface temperature trends”. In: Geophysical Research Letters 45.2 (2018), pp. 1086-1097.

[32] Claire E Bulgin, Christopher J Merchant, and David Ferreira. "Tendencies, variability and persistence of sea surface temperature anomalies”. In: Scientific reports 10.1 (2020), pp. 1-13.

[33] F Alexander Haumann et al. "Sea-ice transport driving Southern Ocean salinity and its recent trends". In: Nature 537.7618 (2016), pp. 89-92.

[34] Ryan P Abernathey et al. "Water-mass transformation by sea ice in the upper branch of the Southern Ocean overturning". In: Nature Geoscience 9.8 (2016), pp. 596-601.

[35] Ivana Cerovečki et al. "The effects of enhanced sea ice export from the Ross Sea on recent cooling and freshening of the southeast Pacific". In: Journal of Climate 32.7 (2019), pp. 2013-2035.

[36] B Buongiorno Nardelli et al. "Southern Ocean mixed-layer seasonal and interannual variations from combined satellite and in situ data". In: Journal of Geophysical Research: Oceans 122.12 (2017), pp. 10042-10060.

[37] Thomas L Frölicher et al. "Dominance of the Southern Ocean in anthropogenic carbon and heat uptake in CMIP5 models". In: Journal of Climate 28.2 (2015), pp. 862-886.

[38] Jia-Rui Shi, Shang-Ping Xie, and Lynne D Talley. "Evolving relative importance of the Southern Ocean and North Atlantic in anthropogenic ocean heat uptake". In: Journal of Climate 31.18 (2018), pp. 7459-7479.

[39] David Ferreira et al. "Antarctic Ocean and sea ice response to ozone depletion: A two-time-scale problem". In: Journal of Climate 28.3 (2015), pp. 1206-1226.

[40] Ivana Cerovečki and Matthew R Mazloff. "The spatiotemporal structure of diabatic processes governing the evolution of Subantarctic Mode Water in the Southern Ocean". In: Journal of Physical Oceanography 46.2 (2016), pp. 683-710.

[41] Libao Gao, Stephen R Rintoul, and Weidong Yu. "Recent wind-driven change in Subantarctic Mode Water and its impact on ocean heat storage". In: Nature Climate Change 8.1 (2018), pp. 58-63.

[42] Nikolaos Skliris et al. "Salinity changes in the World Ocean since 1950 in relation to changing surface freshwater fluxes". In: Climate dynamics 43.3-4 (2014), pp. 709-736.

[43] Martha W Buckley and John Marshall. "Observations, inferences, and mechanisms of the Atlantic Meridional Overturning Circulation: A review”. In: Reviews of Geophysics 54.1 (2016), pp. 5-63.

[44] Eleanor Frajka-Williams et al. "Atlantic meridional overturning circulation: Observed transport and variability". In: Frontiers in Marine Science 6 (2019), p. 260.

[45] Levke Caesar et al. “Current Atlantic Meridional Overturning Circulation weakest in last millennium”. In: Nature Geoscience (2021), pp. 1-3.

[46] Ben I Moat et al. "Pending recovery in the strength of the meridional overturning circulation at $26^{\circ} \mathrm{N}$ ". In: Ocean Science 16.4 (2020), pp. 863-874.

[47] E Frajka-Williams et al. "Coherent circulation changes in the Deep North Atlantic from $16 \mathrm{~N}$ and $26 \mathrm{~N}$ transport arrays". In: Journal of Geophysical Research: Oceans 123.5 (2018), pp. 3427-3443.

[48] Scott C Doney et al. "Ocean acidification: the other CO2 problem". In: Annual review of marine science 1 (2009), pp. 169-192. 

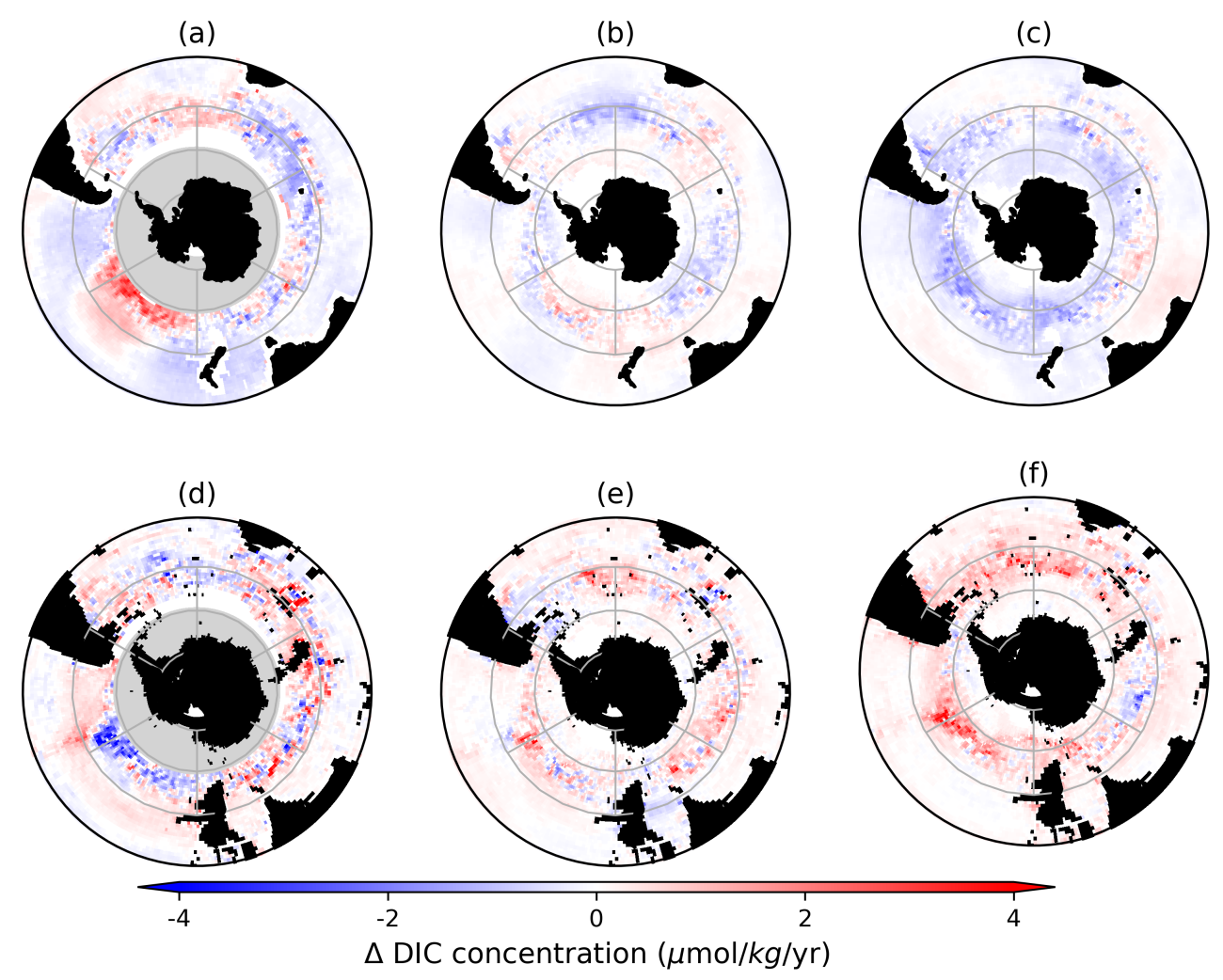

Figure 1: Linear trends in DIC concentration. (a-c) averaged over top $1 \mathrm{~km}$, (d-f) averaged over $2-4 \mathrm{~km}$ depth. Values are calculated over: (left) 1993-1999, (middle) 2000-2009, and (right) 2010-2019. Linear trends outside the $5 \%$ significance level $(p \geq 0.05)$ are excluded. Areas shaded in grey indicate regions of insufficient data for trend calculations. 
(a)

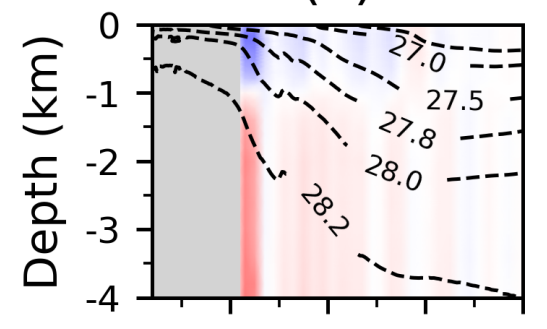

(d)

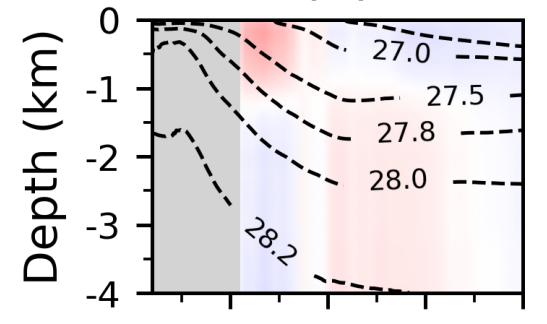

(g)

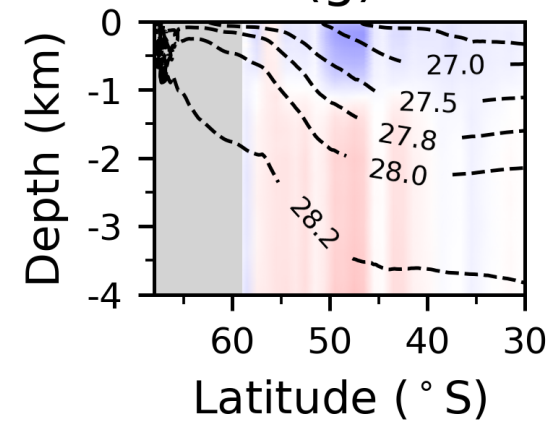

(b)

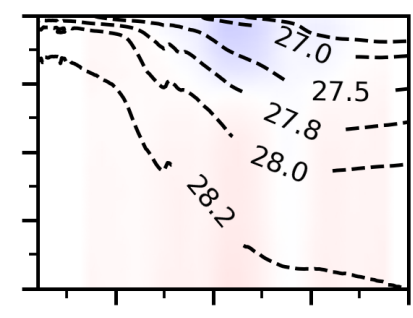

(e)

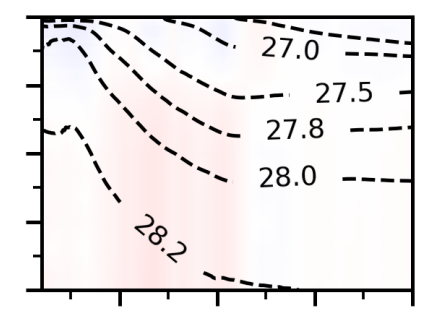

(h)

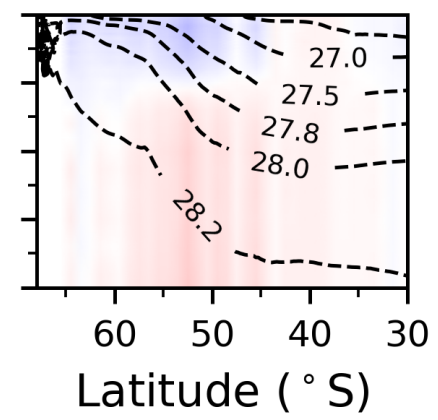

(c)

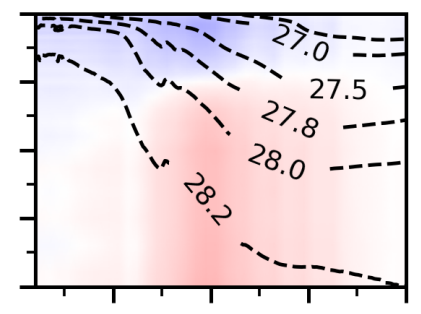

(f)

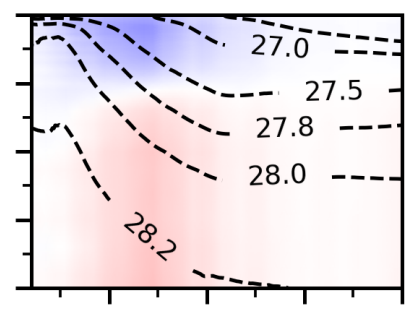

(i)

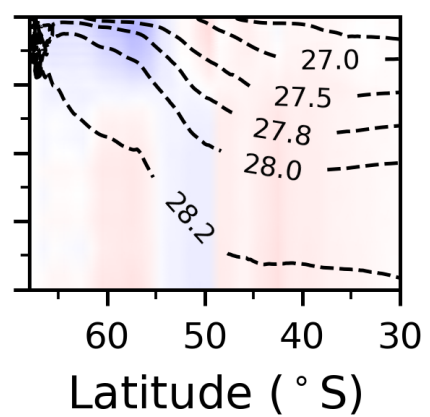

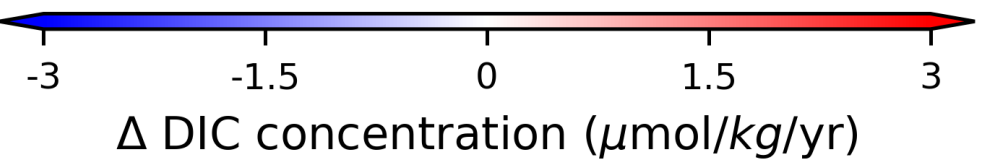

Figure 2: Linear trends in DIC concentration with depth: (left) 1993-1999, (middle) 2000-2009, (right) 20102019. Zonal means of (a-c) Atlantic, (d-e) Pacific, and (g-i) Indian Oceans. Black dashed contours correspond to isosurfaces of neutral density $\gamma_{N}$ from B-SOSE averaged zonally and temporally over 2008-2012 (unlabeled contour: $\left.\gamma_{N}=26.6 \mathrm{~kg} / \mathrm{m}^{3}\right)$. 


\section{Deep-learning model}

The deep-learning model, which is a type of a neural network, used in this study was adapted from the Recurrent U-net introduced in a previous study aimed to predict atmospheric ozone concentrations ${ }^{15}$. The schematic diagram of the model is shown in Figure 3. The model consists of both convolutional neural networks (CNNs) and recurrent neural networks (RNNs) $)^{49}$. The first 3 convolutional blocks are used as an encoder, which transforms the input information into high-dimensional feature space using trainable parameters. Trainable parameters in each convolutional block include the weights and biases in the fully-connected layers ${ }^{50}$ and the convolutional kernels in the convolutional layers. The output from the $3 \mathrm{rd}$ convolutional block is then forwarded into a long short-term memory ${ }^{51}$ (LSTM) cell to capture temporal dynamics in the features. After the LSTM cell, the relationship between these features and the output variables is captured by a decoder, which contains another 3 convolutional blocks with descending depths. Three residual learning connections are added between the encoder and the decoder parts, in order to stabilize the training and capture more direct relationship between input and output variables ${ }^{52}$. We used the mean squared error loss function to train the deep-learning model on a NVIDIA T4 Tensor graphics processing unit (GPU). The Adam optimization algorithm was applied to boost the speed of training ${ }^{53}$.

In this model, we used sea surface temperature, sea surface height, ocean surface velocities, $10 \mathrm{~m}$ wind speeds, total heat flux at the ocean surface, ocean surface chlorophyll-a, and ocean surface partial pressure of $\mathrm{CO}_{2}$ as input variables (predictors). The developed model predicts dissolved inorganic carbon (DIC) concentrations south of $30^{\circ} \mathrm{S}$ in the upper $4 \mathrm{~km}$ of the ocean. These input variables attempted to capture physical (e.g., ocean circulation and mixing), biological (e.g., uptake of $\mathrm{CO}_{2}$ by photosynthetic organisms), and chemical (e.g., uptake of atmospheric $\mathrm{CO}_{2}$ at ocean surface) processes that may affect DIC distribution. While there are many other factors (e.g, sinking rates of organic matter, organic matter remineralization rates, total alkalinity, calcification) that could change DIC concentrations, we chose variables that could be easily measured at the ocean surface, such that the measurements better constrained and available at higher spatial and temporal resolutions than measurements in the ocean interior. We trained the deeplearning model in two phases: first augmenting the volume of data using a biogeochemical ocean circulation model, and then correcting for biases of this model using observational data. We detail the datasets that we used and each of the training phases in the following sections.

Ocean carbon sink has been previously estimated using different methods. However, these methods may either produce indirect bulk estimates over an entire ocean basin (i.e., inverse models ${ }^{54}$ ), be numerically expensive (i.e. ocean circulation models ${ }^{14}$ ), or have limited temporal coverage (i.e., interpolations of direct measurements ${ }^{5,6}$ ). Our deep-learning model attempted to address these issues. In our model, because of the high spatio-temporal availability of the satellite-based input variables, we were able to create a dataset of DIC concentrations at $1^{\circ}$ horizontal resolution in the upper $4 \mathrm{~km}$ of the ocean at 5 -day intervals between $1993-2019$. It allowed us to create a timeseries and compute DIC trends at each individual grid cell over this time period. As a result, we were able to explore spatial patterns in temporal trends, rather than only comparing aggregate decadal averages as in previous studies ${ }^{5,6}$. Using neural networks is also advantageous, as they can capture non-linear relationships between the predictor variables, in contrast to the linear regression models used in previous studies ${ }^{5}$. In addition, this deep-learning model can compute DIC concentrations over the entire Southern Ocean domain very quickly (order of $1-2$ T4 GPU computational hours required for one year of DIC calculations), which makes it ideal for future monitoring of the ocean carbon sink using new satellite data as it becomes available. Finally, it is important to note that a previous study ${ }^{20}$ showed that errors of neural network predictions are reduced when the domain is constrained to a single basin rather than the global ocean, and our model was developed and trained specifically over the Southern Ocean basin only.

\section{Data sets}

\section{Biogeochemical Southern Ocean State Estimate (B-SOSE)}

B-SOSE ${ }^{14}$ is a data-assimilating model that incorporates Biogeochemistry with Light, Iron, Nutrients, and Gases model $(B L I N G)^{55}$ into a data-constrained general circulation model of the Southern Ocean (SOSE) $)^{56}$. The model has uniform horizontal resolution of $1 / 3^{\circ}$ over $30-78^{\circ} \mathrm{S}$; spacing of 52 vertical layers varies with depth from $4.2 \mathrm{~m}$ near the surface to $400 \mathrm{~m}$ in the deepest layers. The output data contains both physical (e.g., temperature, salinity, flow velocity) and biogeochemical (e.g., concentrations of DIC, dissolved oxygen, pH, and chlorophyll-a). It is available at 3-day intervals over 2008-2012 period. The biogeochemical portion of the model includes carbon, nitrogen, and phosphorus cycling, phytoplankton population dynamics, and iron chemistry. The model assimilates in-situ observational data of the carbon system, oxygen, and nutrients from bgc-Argo, GLODAPv2 ${ }^{17}$, and Surface Ocean CO2 product version 4 $(\mathrm{SOCATv} 4)^{57}$ in addition to physical constraints from hydrographic and satellite observations.

\section{Satellite-based products}

We used data from the following sets produced based on satellite observations. All data was available over between $1993-2019$ over the Southern Ocean (i.e. south of $30^{\circ} \mathrm{S}$ ), with the exception of chl-a, which was only available north of $60^{\circ} \mathrm{S}$.

- Horizontal ocean surface velocities $(u, v)$ were obtained from Ocean Surface Current Analysis Real-time (OSCAR $)^{a}$, which uses satellite sea surface height, wind, and temperature for computations ${ }^{58}$. Data are available at $1 / 3^{\circ}$ and 5 -day resolution between $1992-2020$.

- Sea surface height (SSH) was obtained from Copernicus Marine Environment Monitoring Service (CMEMS) dataset $^{b}$ that merges altimetry data from available missions for a more consistent and homogeneous product. It

${ }^{a}$ https://podaac.jpl.nasa.gov/dataset/OSCAR_L4_OC_third-deg

${ }^{b}$ https://resources .marine.copernicus.eu/, dataset: SEALEVEL_GLO_PHY_L4_REP_OBSERVATIONS_008_047 
is available $1 / 4^{\circ}$ and 5 -day resolution between $1993-2020$. $\mathrm{SSH}$ was used to compute vertical velocity $(w)$ at the ocean surface to be consistent with calculations in B-SOSE.

- Zonal and meridional components of $10 \mathrm{~m}$ wind speed, sea surface temperature (SST), and total heat flux at the ocean surface were obtained from ERA $5^{59}$, which is a comprehensive reanalysis dataset that assimilates available observations in the upper air and near surface. Data $^{c}$ is available at an hourly temporal resolution and $31 \mathrm{~km}$ spatial resolution from 1979 - 2020. Total heat flux was computed as the sum of net shortwave and longwave radiation and sensible and latent heat, using the hourly accumulation values (in $J / \mathrm{m}^{2}$ ) converted to flux units $\left(W / m^{2}\right)$

- Surface chlorophyll-a (chl-a) concentrations were obtained from GlobColour dataset ${ }^{d}$ by the European Space Agency, which merges data from four satellite sources. Data used here is available at $1 / 4^{\circ}$ and $8-$ day resolution from $1997-2020$.

- An estimate from neural network ${ }^{16}$ was used for surface partial pressure of $\mathrm{CO}_{2}\left(p \mathrm{CO}_{2}\right)$. This neural network uses primarily satellite observations as inputs to interpolate the available shipboard measurements of $p \mathrm{CO}_{2}$ over $1^{\circ}$ grid at a monthly resolution from $1982-2020$. Using this neural network-based dataset is advantageous compared to simply spatially-interpolated observations because it accounts for spatial and temporal heterogeneity of observational data availability.

\section{Observational DIC data}

We trained the model with DIC data from two observational datasets. The first one was GLobal Ocean Data Analysis Project Version 2 (GLODAPv2) ${ }^{17,18}$, which is a compilation of inorganic carbon data collected during research cruises. We used in-situ data from the original shipboard measurements rather than a globally remapped product. The second dataset was collected by Southern Ocean Carbon and Climate Observations and Modeling project (SOCCOM) ${ }^{e}$ Argo floats equipped with biogeochemical sensors. Here we only use data with "good" quality flag. We used GLODAPv2 shipboard measurements available between 1998 - 2019 and Argo float measurements available between 2014 2019. Over the period where the two datasets overlap, the number of Argo float measurements was much larger than that of the shipboard measurements (cf. Extended Data Fig. 4). Argo float data also had better temporal coverage, whereas wintertime shipboard measurements were limited ${ }^{20}$. However, data from Argo floats was only available above $2 \mathrm{~km}$ depth, whereas there were shipboard measurements below this depth, though far less numerous than above (cf. Extended Data Fig. 4). Furthermore, it has been shown using both Argo float and shipboard measurements in neural network training minimizes the root mean square error between the model predictions and observations ${ }^{20}$, so we used both datasets for training our model.

\section{Model training}

The high spatial and temporal resolutions of B-SOSE over a three-dimensional domain made it a good training set for a deep-learning model. B-SOSE data was also more evenly distributed spatially and temporally than the observations. In particular, it had significantly more data points available below $2 \mathrm{~km}$, where observations were especially sparse. Thus, including B-SOSE dataset into training was important to prevent overfitting of the deep-learning model to the observational data. To correct for any inherent errors of the B-SOSE model and to account for its short availability period (only 5 years), it was also necessary to further train a model with observed data (i.e. shipboard and Argo float measurements). However, because of the vast difference in the number of available data points between B-SOSE ( $\sim 10$ million data points per timestep over 609 timesteps) and observations $(\sim 450,000$ data points in total), it was necessary to train the model in two phases; otherwise, the deep-learning model output would have been heavily biased towards B-SOSE. Finally, because the near-coastal processes in shallow waters may be significantly different from the dynamics of the open ocean, we excluded regions with less than $1 \mathrm{~km}$ depth from our model training.

\section{Phase 1}

In the first training phase of the deep-learning model, we used SSH, ocean surface velocities $(u, v, w)$, ocean surface heat flux, $p \mathrm{CO}_{2}$, and chl-a concentrations from B-SOSE output and SST and $10 \mathrm{~m}$ wind speed velocities from ERA5. We chose to use these two predictors from ERA5 rather than B-SOSE output because of the higher spatio-temporal resolution of the ERA5 data, which would be advantageous for matching to the in-situ measurements in Phase 2 of the model training. The hourly ERA5 data was averaged over 3 -day period to have the same temporal resolution as B-SOSE. DIC concentrations from B-SOSE were taken as the target for model training. We used data over $2008-2011$ period for model training, while reserving a randomly-sampled $10 \%$ of it for in-sample validation to prevent overfitting. The 2012 data was then used as out-of-sample validation set for the model.

The comparison with model-predicted DIC from Phase 1 training and B-SOSE DIC are shown in Extended Data Fig. 5, averaged over $1 \mathrm{~km}$ depth intervals over the year 2012, which we chose as the testing period for the model. The relative errors were overall less than $\pm 5 \%$ within each depth interval; the errors did, however, increase with depth, where fewer data points were available because of the larger vertical grid spacing and the range of DIC concentration values was not as large compared to the surface. In the deep layers (below $2 \mathrm{~km}$ depth), the model overestimated DIC concentrations in the Atlantic sector and underestimated them in the Pacific sector north of $45^{\circ} \mathrm{S}$ with relative errors much larger than in the poleward regions. Northward of these latitudes, exchange with other ocean basins plays an important role in the deep circulation, and it is possible that local surface and near-surface properties were not sufficient to capture the inter-basin transport dynamics ${ }^{20}$. As such, we were careful in not discussing DIC trends in this part of the ocean in text.

chttps://cds.climate.copernicus.eu/cdsapp\#!/home

${ }^{d}$ https://www.globcolour.info/products_description.html

${ }^{e}$ https://soccom.princeton.edu/ 
The relative errors were centered and symmetrically distributed around approximately zero at all depths (cf. Extended Data Fig. 6). The errors showed overall no systematic bias towards high or low values, though there were more high-end outliers compared with low-end outliers below $2 \mathrm{~km}$. The spread (including the outliers) was larger in the upper $1 \mathrm{~km}$, possibly related to a greater degree of noise associated with small-scale near-surface processes that was more difficult to capture with the model. Horizontally-averaged profile of model-predicted DIC concentration also showed very small deviation (less than $0.2 \%$ from B-SOSE data across different depth levels (cf. Extended Data Fig. 7)).

The heatscatter plot of DIC concentrations predicted by the deep-learning model over the three-dimensional domain for 2012 is shown in Extended Data Fig. 8 in comparison with B-SOSE DIC concentrations. The vast majority of the points were along the one-to-one line with a high linear correlation coefficient $\left(r^{2}=0.98\right)$ between the model-predicted and B-SOSE DIC concentrations. The offset of the linear correlation was also small ( $45 \mu \mathrm{mol} / \mathrm{kg})$, especially given that DIC concentrations were mostly within the $2050-2350 \mu \mathrm{mol} / \mathrm{kg}$ range.

\section{Phase 2}

In the second training phase, we applied the deep-learning model obtained from Phase 1 to the previously-described satellite-based observational data and further trained the model to minimize the RMSE between the model predictions and shipboard and Argo float measurements. When chl-a measurements were not available (primarily due to presence of sea ice), values within those cells were set to zero to be consistent with B-SOSE instead of setting it to a non-zero minimum chl-a concentration value like in some previous $p \mathrm{CO}_{2}$ models ${ }^{60}$. The observational DIC data was re-mapped to the same depth levels as the B-SOSE dataset to be consistent with Phase 1 training output. We used the most recent $20 \%$ of the observational data as an out-of-sample test dataset, and the rest as the training dataset. Again, a randomlysampled $10 \%$ of the training set was used for in-sample testing. To compare the two observational DIC datasets, we trained the model with (1) only shipboard data, and (2) with shipboard and Argo float data.

The distributions of relative errors of the model prediction (cf. Extended Data Fig. 9) were again mostly symmetric around zero with most values falling between $\pm 3 \%$. The outliers could be the result of both model prediction errors and the variability in data collection from different cruises and any systematic differences between shipboard and Argo float measurements. As expected, the correlation between predicted and observed DIC concentration values improved when the model is trained with more data points by including the Argo float measurements (compare Extended Data Fig. 10a,b). When the model was trained with both shipboard and Argo float data, considerably more model-predicted points fell along the one-to-one line. This result is consistent with previous analysis of neural networks used for to compute $p \mathrm{CO}_{2}$, concluding that both shipboard and Argo float data were necessary for more accurate model predictions ${ }^{20}$. However, because of the much more limited number of observations compared with the number of available B-SOSE data points, the linear fit (e.g.,correlation coefficient) was worse compared with Phase 1 training (cf. Extended Data Fig. 8). This demonstrated that performance of a deep-learning model improved with more data points available for training and why it was important to pre-train the model with a large volume of B-SOSE data in Phase 1.

\section{Linear decadal trend estimations}

We applied the trained deep-learning model to the satellite-based observational datasets to compute DIC concentrations at $1^{\circ}$ horizontal resolution and at the same vertical levels used in the B-SOSE model. DIC concentrations were computed at a 5 -day resolution over the period between 1993 - 2019, for which the input variables were available. Chlorophyll-a concentrations were only available after 1997 and for the prior years, climatologically-averaged chl-a concentrations computed over $1997-2019$ were used. Same technique was applied to a previous neural network predicting $p \mathrm{CO}_{2}{ }^{3}$. We then divided the obtained DIC concentration data into three decadal time periods: 1993-1999, 2000-2009, and 2010-2019. This division was useful in comparing the evolution of linear trends across different sectors of the Southern Ocean and relating our results to the previous findings of a weakening trend of the Southern Ocean carbon sink in the $1990 \mathrm{~s}^{10,11}$ and a strengthening trend in the $2000 \mathrm{~s}^{3,4}$.

Timeseries over each decadal segment were then extracted at each (latitude,longitude,depth) grid cell. In order to fill in the missing data points in the timeseries, which could result from of ice or cloud cover or other problems with observational data, we used cubic-spline interpolation. However, to prevent over-interpolation at a location where too much data was missing, we applied criteria used in a previous study for gap-filling ocean-carbon data ${ }^{61}$. Namely, we restricted the interpolations to locations were data was available (1) for at least five years over each decadal period to ensure that the timeseries was long enough to capture seasonal and long-term trends, and (2) for at least $2 / 3$ of a year at some point in the timeseries in order to extract seasonal cycles. Once the missing data was reasonably filled, we subtracted the seasonal cycle, which we calculated over each time period individually using the statsmodels ${ }^{f}$ statistical module. Computing seasonal cycle over each decade rather than using a climatological seasonal mean better accounted for any changes in the seasonal cycles over time. Finally, at each grid cell, from the seasonally-detrended data, we computed linear trends over each decadal period using a linear regression model and excluded trends that are not statistically significant (i.e., outside of the $95 \%$ confidence level with $p \geq 0.05$ ). The statistically significant linear trends were then used to produce Fig. 1 and Fig. 2 in the main text.

\section{Data Availability}

Published dataset of DIC concentrations over 1993-2019 period computed by the deep-learning model presented in this study can be found at https://doi.org/10.5683/SP2/FTQYTV.

\section{Code Availability}

Codes for Phase 1 and 2 training and testing of the model and for computing DIC from satellite-based products decribed in Methods can be found at https://github.com/tailonghe/Southern_Ocean_Carbon.

$f_{\text {https: //www.statsmodels.org/ }}$ 


\section{References}

[49] Jürgen Schmidhuber. "Deep learning in neural networks: An overview". In: Neural Networks 61 (Jan. 2015), pp. 85-117. ISSN: 0893-6080. DOI: 10.1016/j . neunet.2014.09.003. URL: http://dx.doi .org/10. $1016 / j$.neunet. 2014.09.003.

[50] S.H. Shabbeer Basha et al. "Impact of fully connected layers on performance of convolutional neural networks for image classification". In: Neurocomputing 378 (Feb. 2020), pp. 112-119. ISSN: 0925-2312. DOI: 10.1016/ j.neucom. 2019.10.008. URL: http://dx.doi.org/10.1016/j.neucom. 2019.10.008.

[51] Sepp Hochreiter and Jürgen Schmidhuber. "Long Short-Term Memory”. In: Neural Comput. 9.8 (Nov. 1997), pp. 1735-1780. ISSN: 0899-7667. DOI: 10.1162/neco.1997.9.8.1735. URL: https : //doi .org/10. $1162 /$ neco.1997.9.8.1735.

[52] Hao Li et al. Visualizing the Loss Landscape of Neural Nets. 2018. arXiv: 1712.09913 [cs . LG].

[53] Diederik P. Kingma and Jimmy Ba. Adam: A Method for Stochastic Optimization. 2017. arXiv: 1412 . 6980 [cs.LG].

[54] Tim DeVries et al. "Decadal trends in the ocean carbon sink". In: Proceedings of the National Academy of Sciences 116.24 (2019), pp. 11646-11651.

[55] Eric D Galbraith et al. "Regional impacts of iron-light colimitation in a global biogeochemical model". In: Biogeosciences 7.3 (2010), pp. 1043-1064.

[56] Matthew R Mazloff, Patrick Heimbach, and Carl Wunsch. "An eddy-permitting Southern Ocean state estimate". In: Journal of Physical Oceanography 40.5 (2010), pp. 880-899.

[57] Dorothee CE Bakker et al. "An update to the Surface Ocean CO 2 Atlas (SOCAT version 2)". In: Earth System Science Data 6.1 (2014), pp. 69-90.

[58] Fabrice Bonjean and Gary SE Lagerloef. "Diagnostic model and analysis of the surface currents in the tropical Pacific Ocean”. In: Journal of Physical Oceanography 32.10 (2002), pp. 2938-2954.

[59] H. Hersbach et al. "ERA5 hourly data on pressure levels from 1979 to present". In: Copernicus Climate Change Service (C3S) Climate Data Store (CDS) (2018). DOI: 10.24381/cds . bd0915c6.

[60] Sayaka Yasunaka et al. "Arctic Ocean CO 2 uptake: an improved multiyear estimate of the air-sea CO 2 flux incorporating chlorophyll a concentrations". In: Biogeosciences 15.6 (2018), pp. 1643-1661.

[61] Steve D Jones et al. "A statistical gap-filling method to interpolate global monthly surface ocean carbon dioxide data". In: Journal of Advances in Modeling Earth Systems 7.4 (2015), pp. 1554-1575.

\section{Acknowledgements}

We acknowledge the support of the Natural Sciences and Engineering Research Council of Canada (NSERC; Grant No. RGPIN-2015-03684). Data were collected and made freely available by the Southern Ocean Carbon and Climate Observations and Modeling (SOCCOM) Project funded by the National Science Foundation, Division of Polar Programs (NSF PLR -1425989 and OPP-1936222), supplemented by NASA, and by the International Argo Program and the NOAA programs that contribute to it. (http://www . argo .ucsd. edu), http://argo.jcommops .org). The Argo Program is part of the Global Ocean Observing System. Computational resources for the SOSE were provided by NSF XSEDE resource grant OCE130007. Authors thank research teams from Argo, CMEMS, GLODAP, GlobColour, ERA5, NOAA OCADS, OSCAR, and SOCCOM for making the data publicly available. Computations were performed at the SHARCNET HPC Consortium, funded by the Canada Foundation for Innovation under Compute Canada.

\section{Author contributions}

V.Z. designed the project and performed linear trend analysis. T.H. and Z.W. developed and trained the deep-learning model under the supervision of V.Z. and N.G. V.Z. wrote the manuscript with input from N.G.

\section{Competing interests}

Authors report no competing interests. 


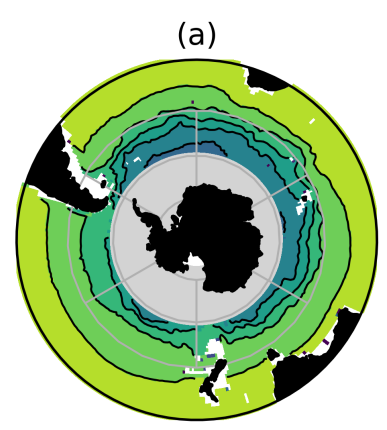

(d)

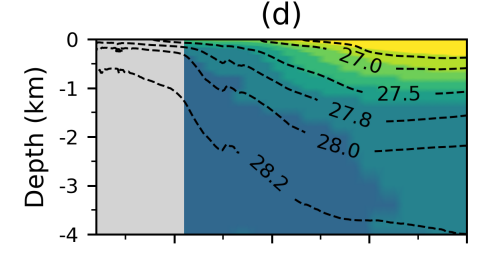

(g)

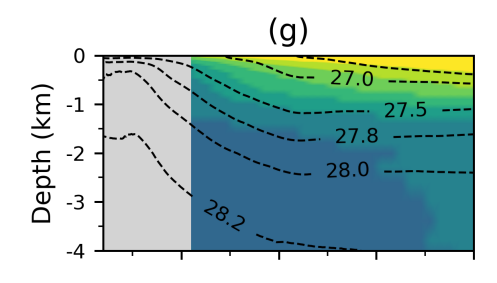

(j)

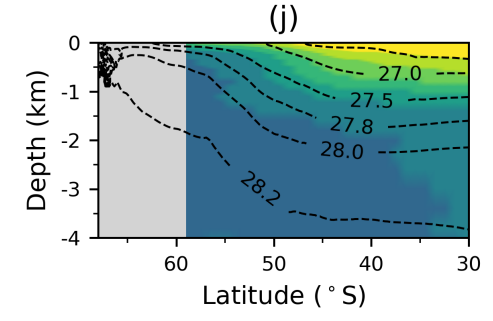

(b)

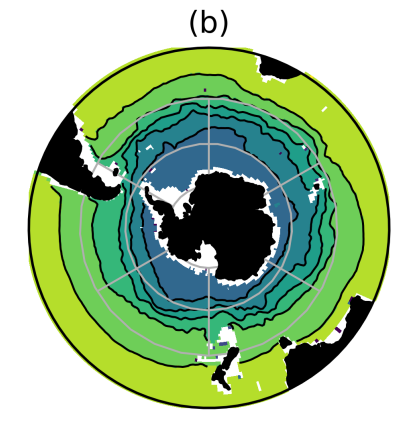

(e)

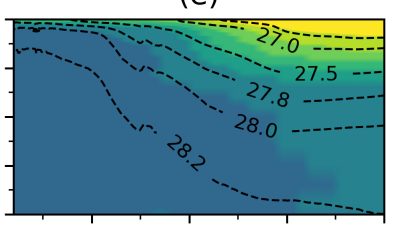

(h)

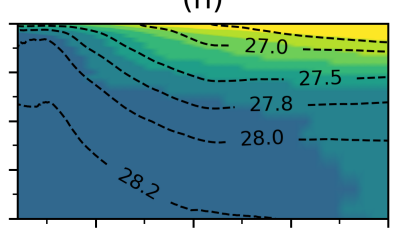

(k)

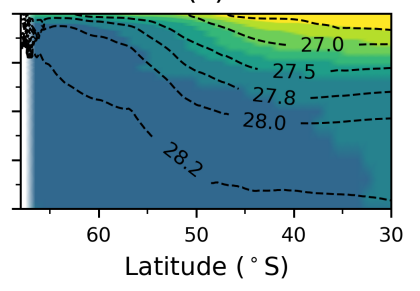

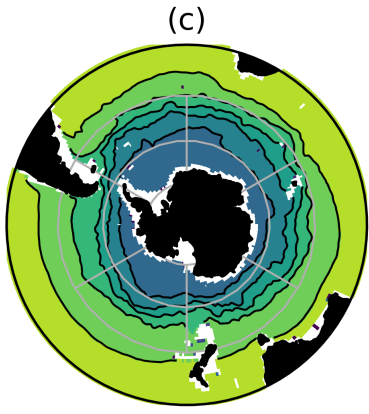

(f)

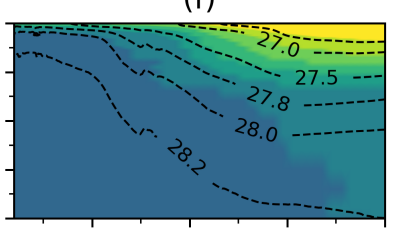

(i)

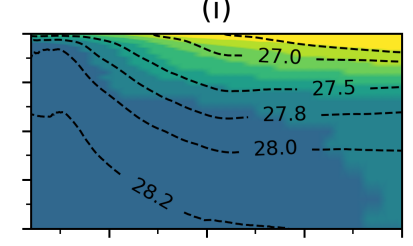

(I)

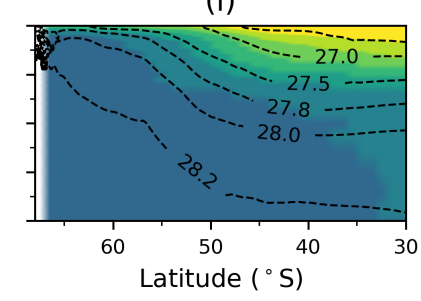

$\begin{array}{lcccc}2100 & 2200 & 2250 & 2350 & 2350 \\ & & \text { DIC concentration }(\mu \mathrm{mol} / \mathrm{kg}) & & \end{array}$

Extended Data Figure 1: DIC concentrations computed using deep learning model: (left) 1993-1999, (middle) 2000-2009, (right) 2010-2019. (a-c) Decadal averages of DIC concentrations over top $1 \mathrm{~km}$ with contours, zonal means of (d-e) Atlantic, (g-i) Pacific, and (j-1) Indian Oceans. Black dashed contours correspond to isosurfaces of neutral density $\gamma_{N}$ from B-SOSE averaged zonally and temporally over $2008-2012$ (unlabeled contour: $\gamma_{N}=26.6 \mathrm{~kg} / \mathrm{m}^{3}$ ). 


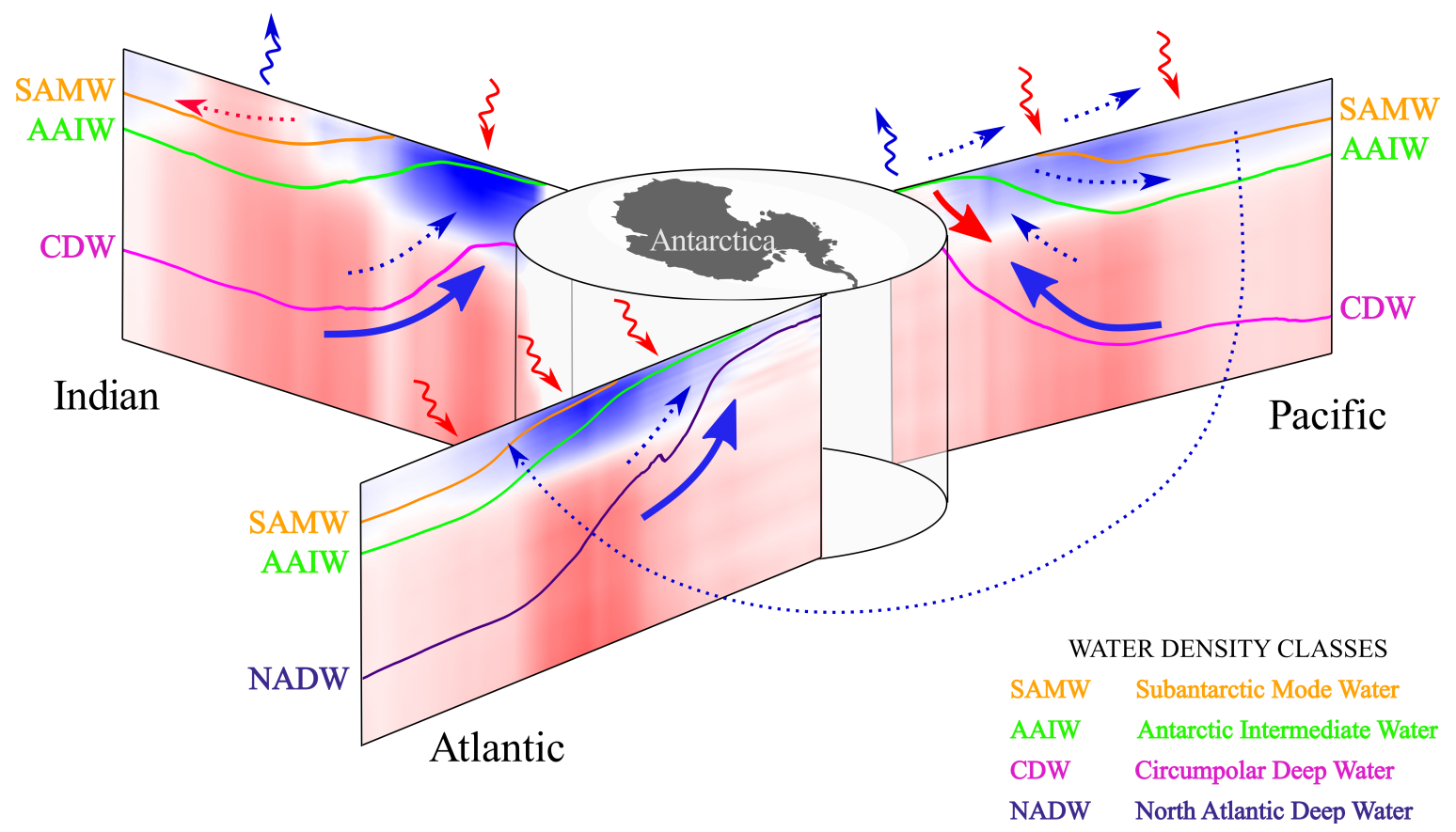

Extended Data Figure 2: Schematic of the mechanisms affecting DIC trends in the 2000s and 2010s between 30 and $75^{\circ} \mathrm{S}$ broken down by ocean sectors. Solid colored lines trace out representative density surfaces of each water-mass (SAMW, AAIW, CDW, NADW). Blue (red) color shading indicates decreasing (increasing) DIC trends over $2000-2019$. Curly arrows mark buoyancy forcing at the surface: blue (red) indicating buoyancy loss, i.e. input of denser water (buoyancy gain, i.e. input of lighter water). Solid thick arrows mark changes in ocean circulation: blue (red) indicating weakening (strengthening) flow in the indicated direction. Small dotted arrows mark relative strength of DIC transport: blue (red) indicating weakening (strengthening) transport or transport of lower (higher) DIC concentrations. 


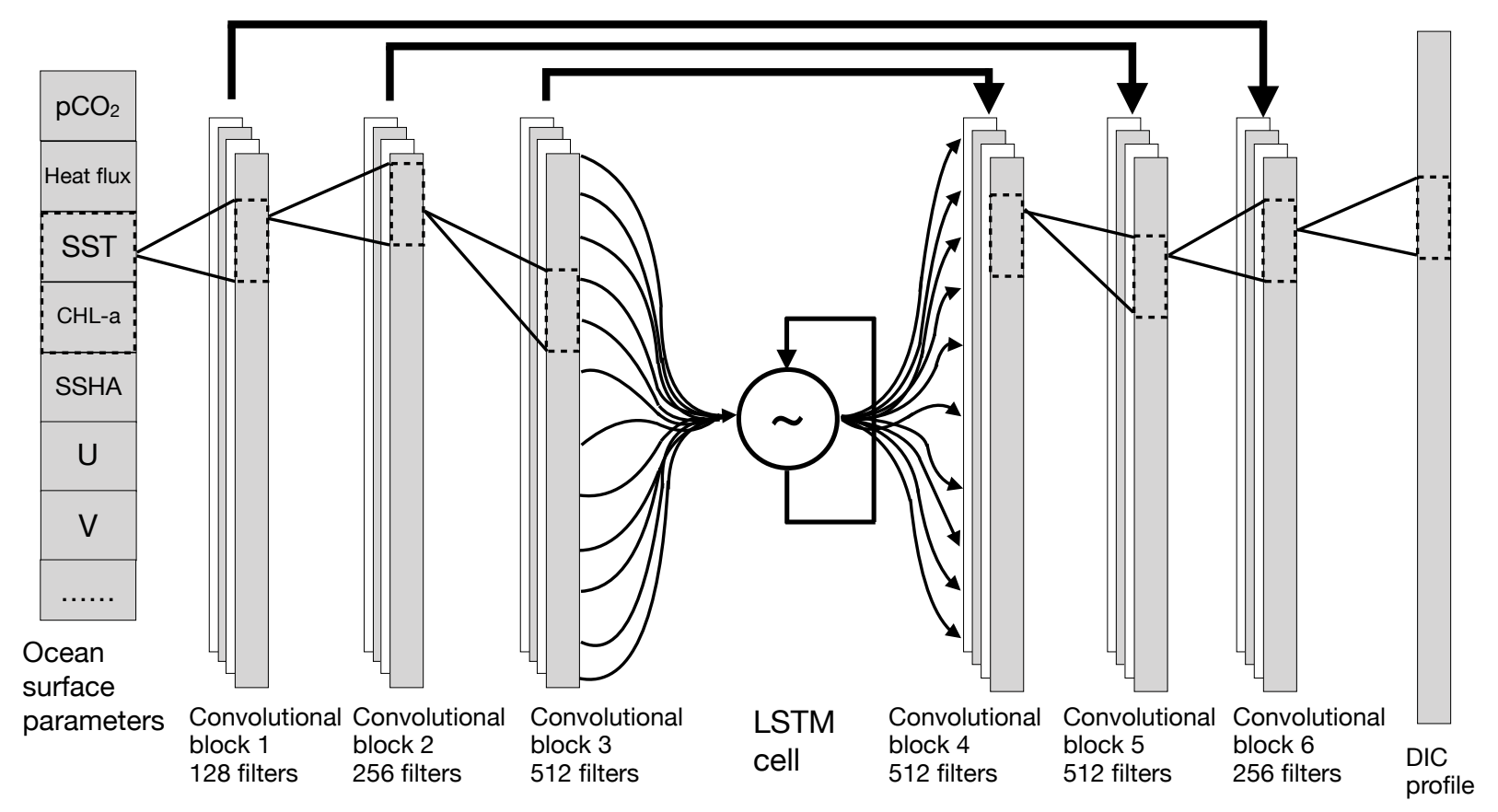

Extended Data Figure 3: Diagram of the one-dimensional recurrent U-net. In each convolutional block, input data is fed through 1 fully-connected layer and then filtered by 2 convolutional layers. 


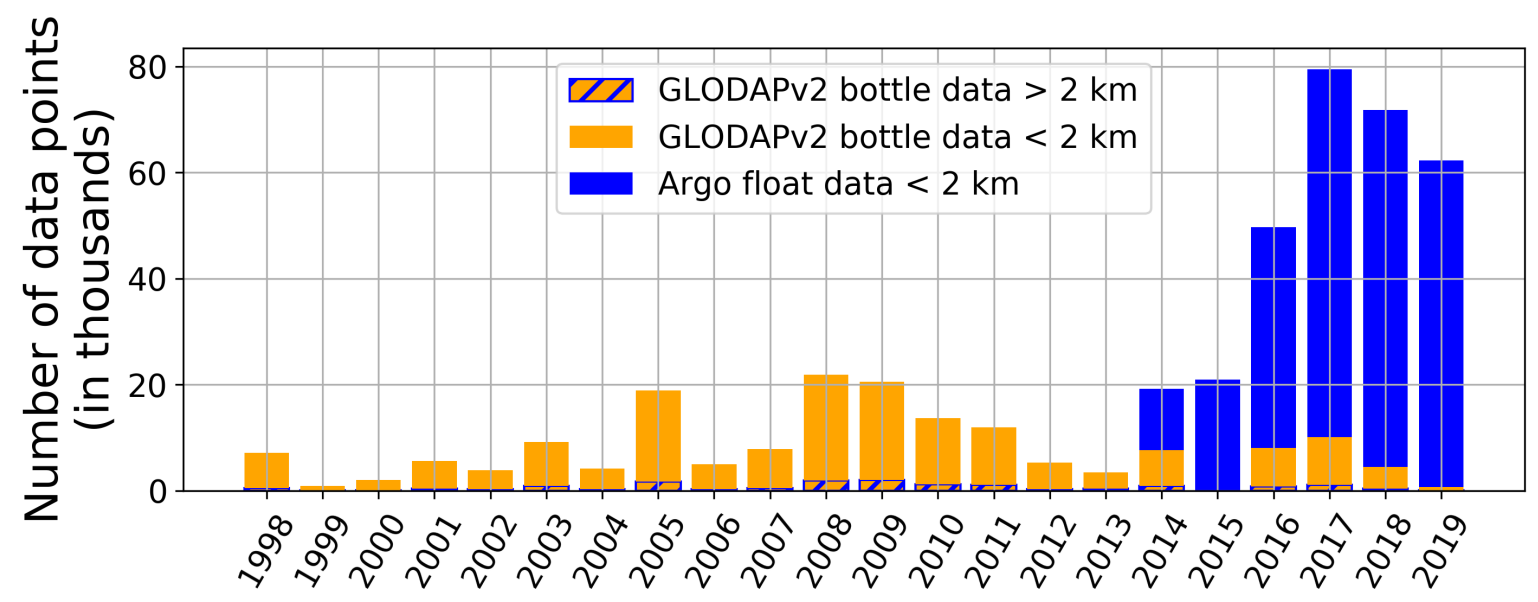

Extended Data Figure 4: Number of interpolated in situ data points in each year between 1998 and 2019 
(a)
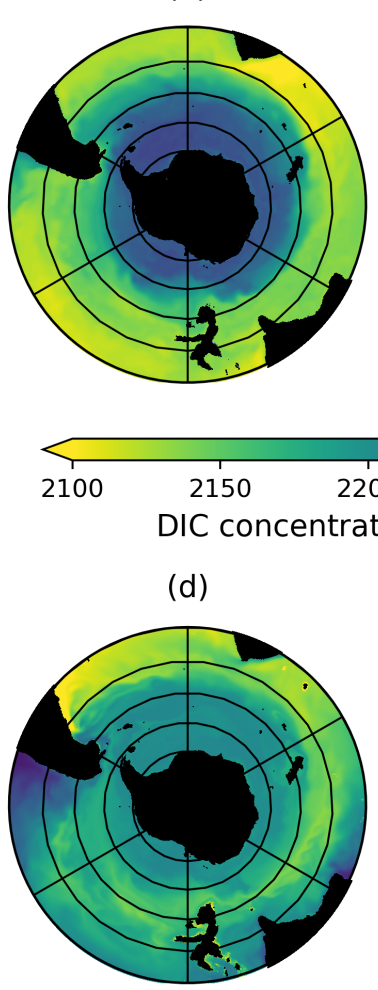

(g)

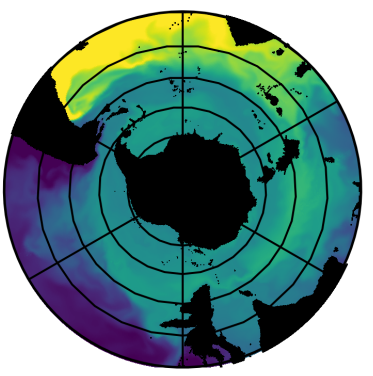

(j)

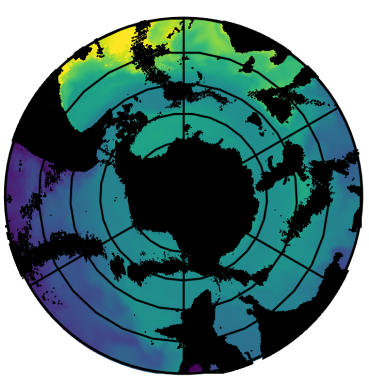

2200 (b)

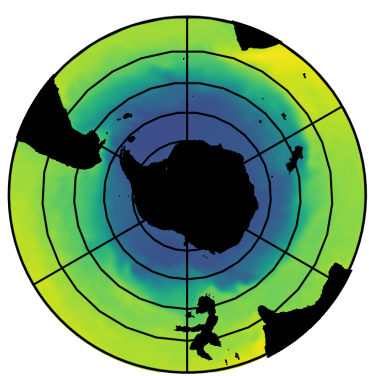

2250

2300

(e)

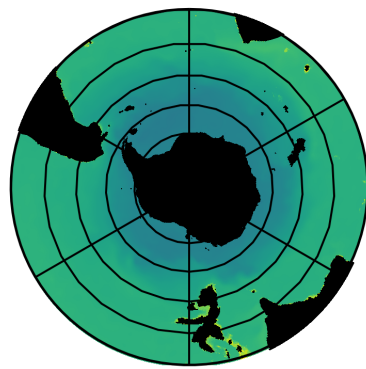

(h)

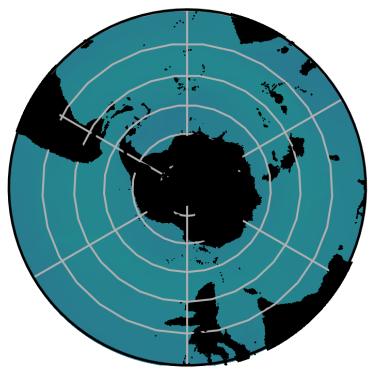

(k)

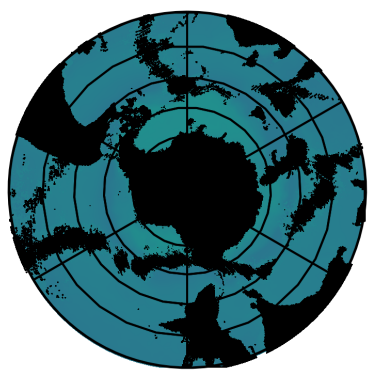

2300

DIC concentration $(\mu \mathrm{mol} / \mathrm{kg})$
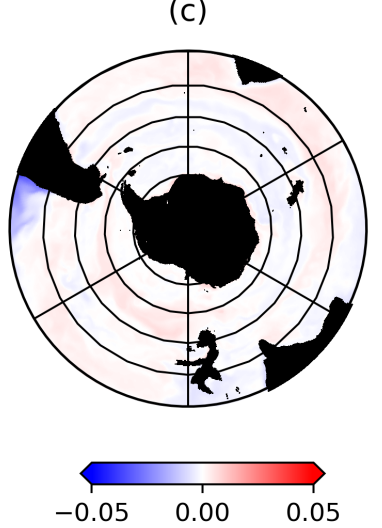

(f)

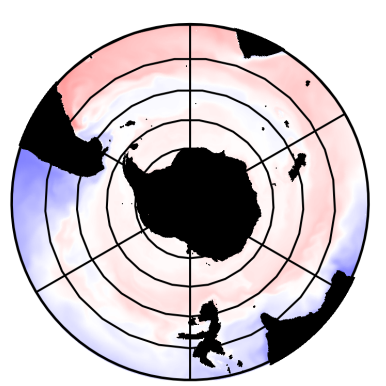

(i)

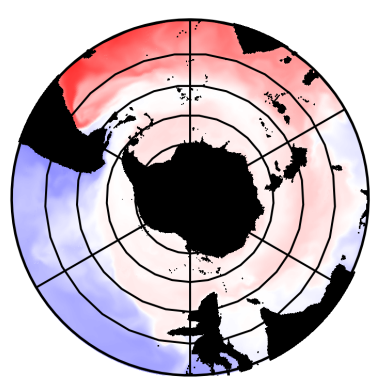

(I)

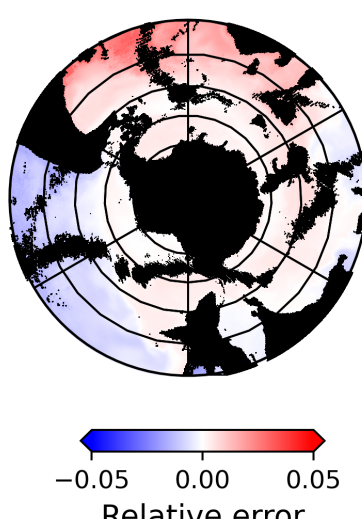

Extended Data Figure 5: DIC concentration vertically averaged over different depth intervals. (left) B-SOSE DIC concentration from B-SOSE averaged over, (middle) DIC concentration predicted by the deep learning model, and (right) relative errors of the deep learning model predictions. All variable are averaged over: (a-c) $0-1 \mathrm{~km},(\mathrm{~d}-\mathrm{f}) 1-2$ $\mathrm{km}$, (g-i) 2-3 km, and (j-i) 3-4 km. 


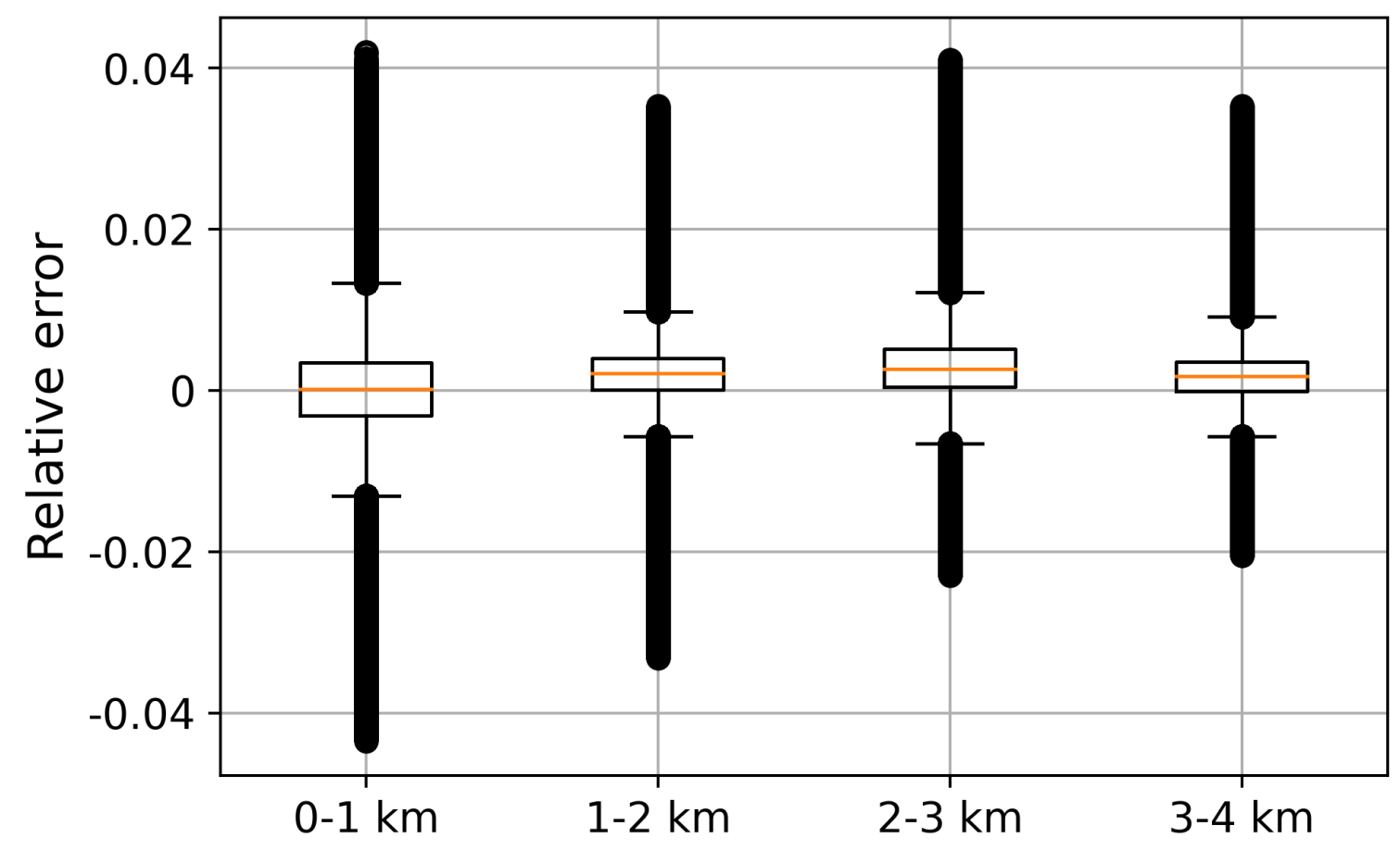

Extended Data Figure 6: Box-and-whiskers plot of relative errors between deep learning model predictions and B-SOSE DIC concentration over different depth intervals. Relative errors are calculated at each B-SOSE grid point, averaged over the test period (year 2012). In box-plots, center line: median; box limits: upper and lower quartiles; whiskers: $1.5 \times$ interquartile range; points: outliers. 
(a)

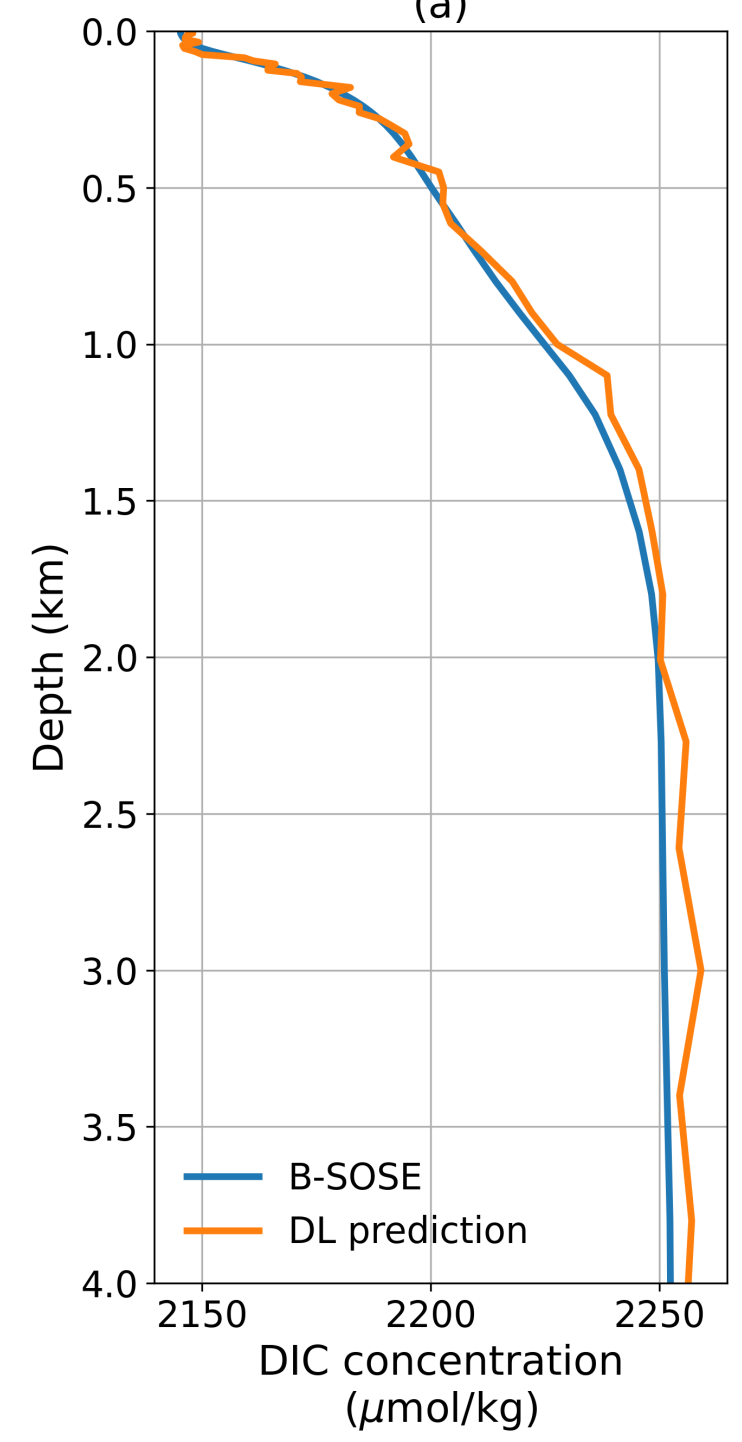

(b)

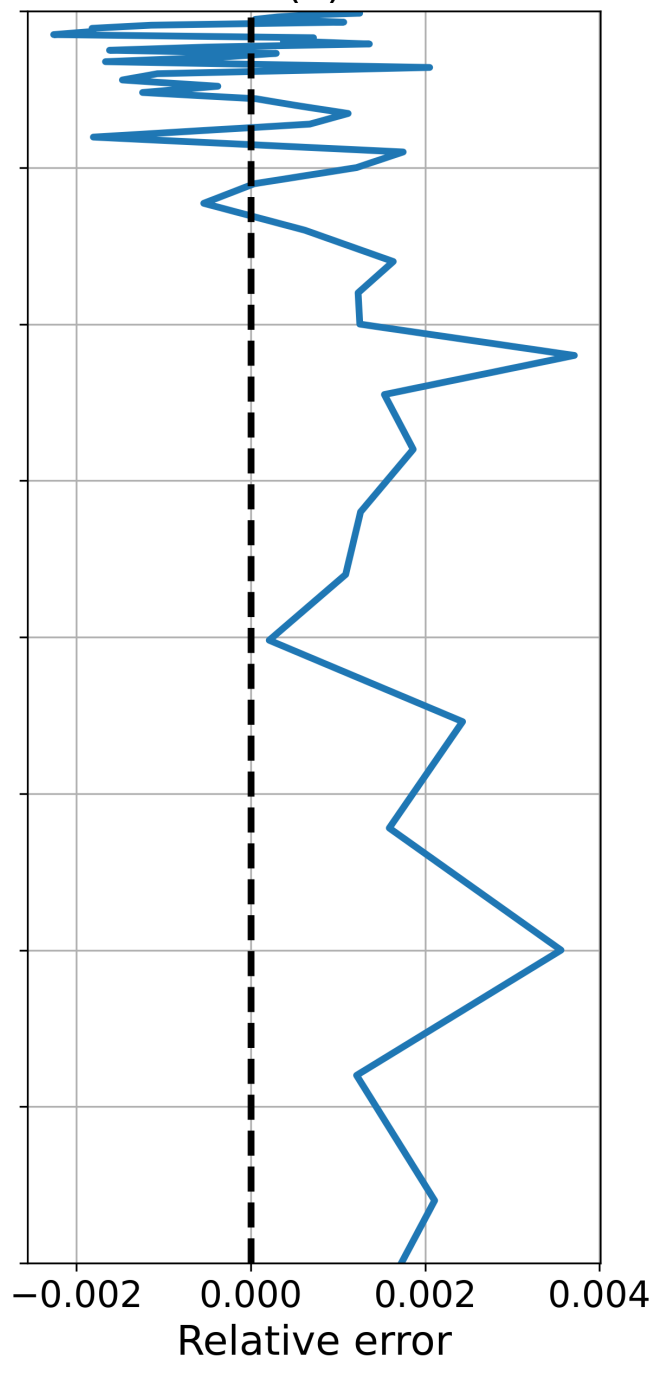

Extended Data Figure 7: Comparison between B-SOSE and deep learning model predicted DIC profiles, averaged within the test period (year 2012). (a) DIC concentration with depth, and (b) horizontally-averaged relative errors with depth. 


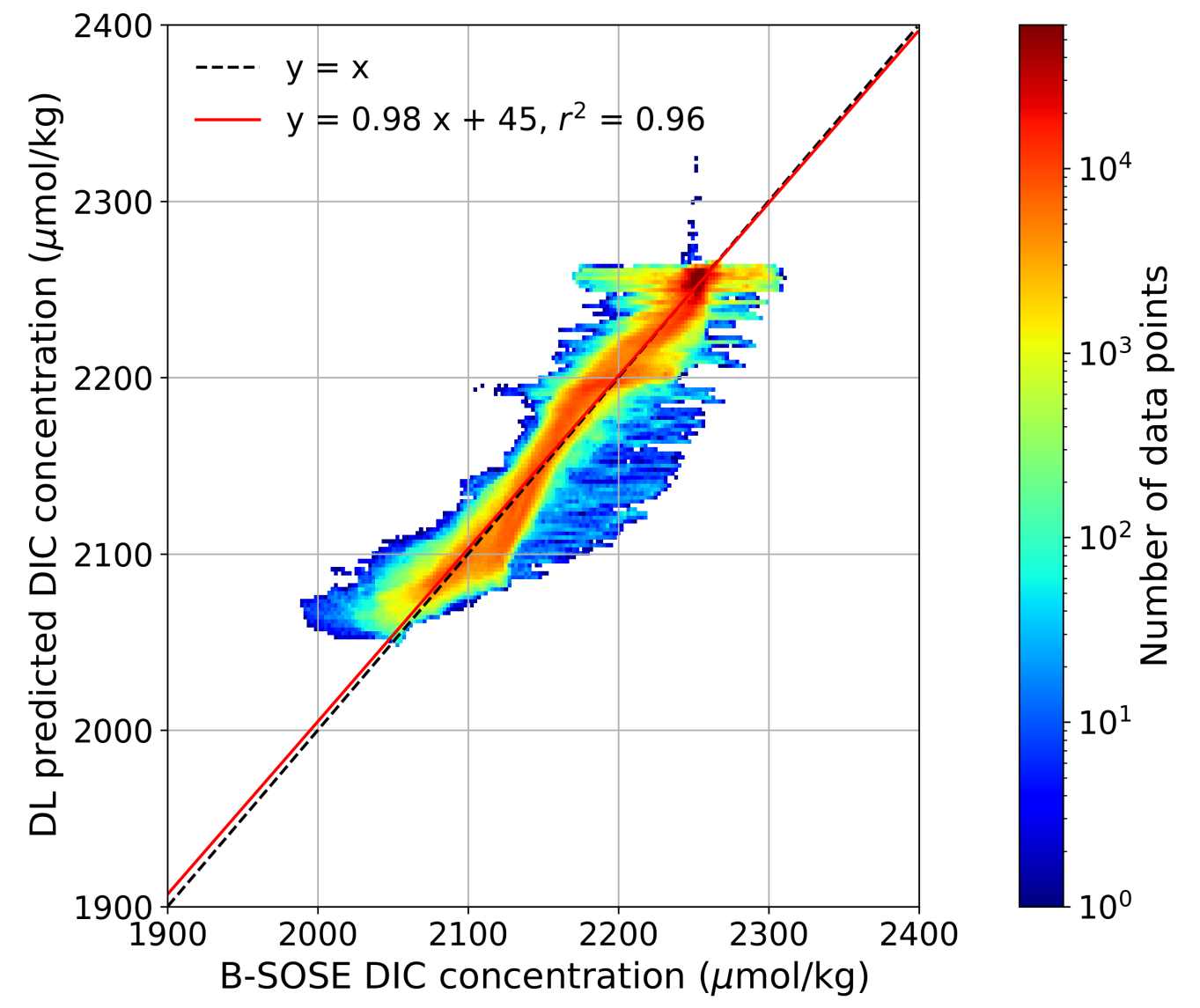

Extended Data Figure 8: Correlation between B-SOSE and deep learning model predicted DIC concentrations. DIC concentration at each B-SOSE grid is temporally averaged over the test period (year 2012). Red lines show the linear fit with $r^{2}$ value reported in the legend compared with one-to-one line in dotted black. 
(a)

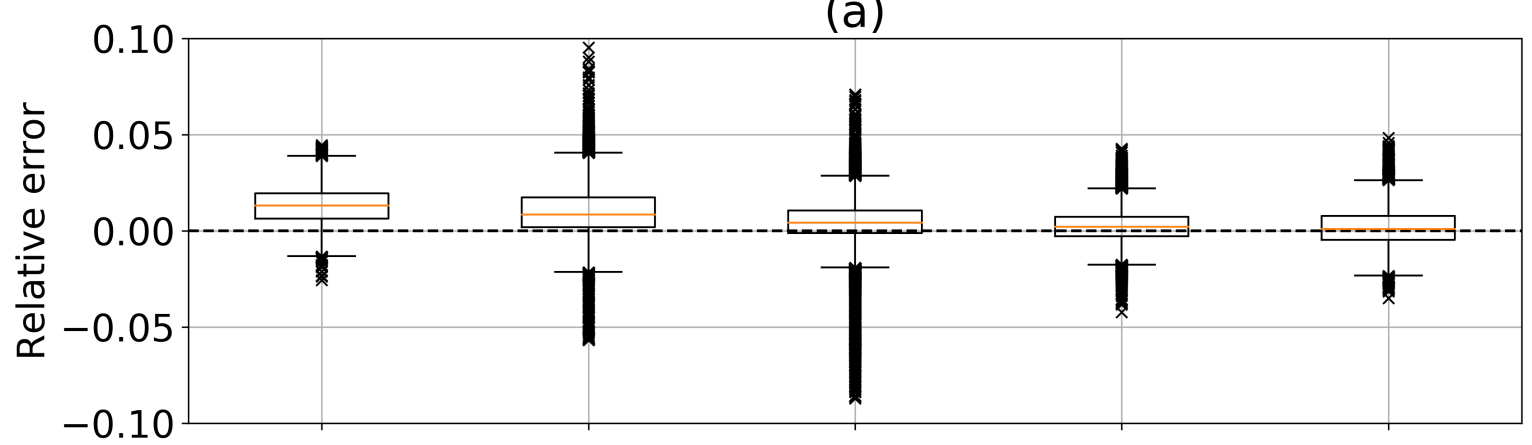

(b)

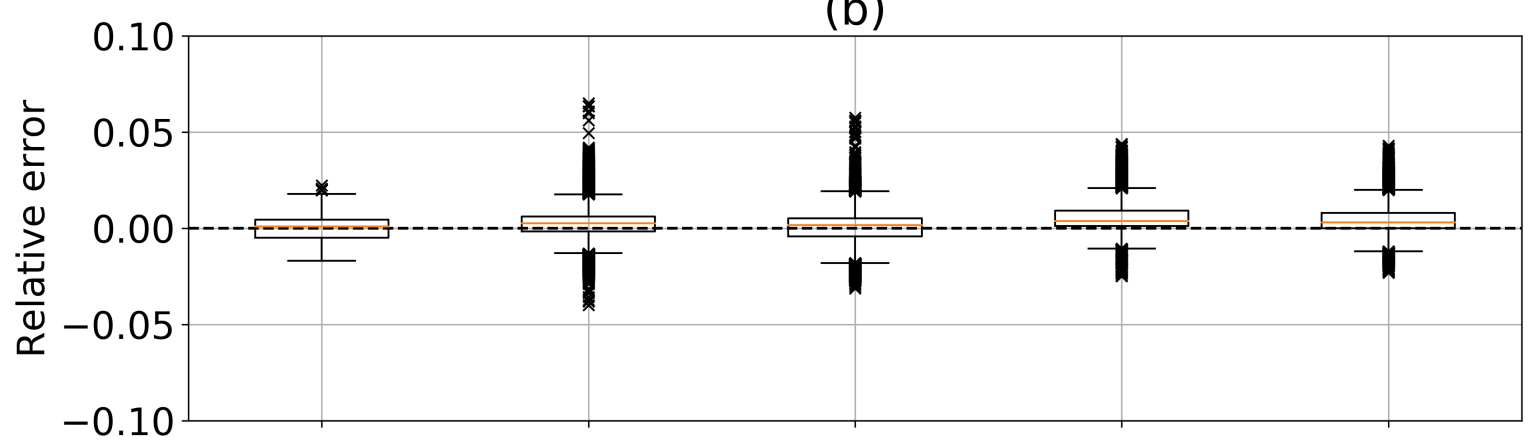

(c)

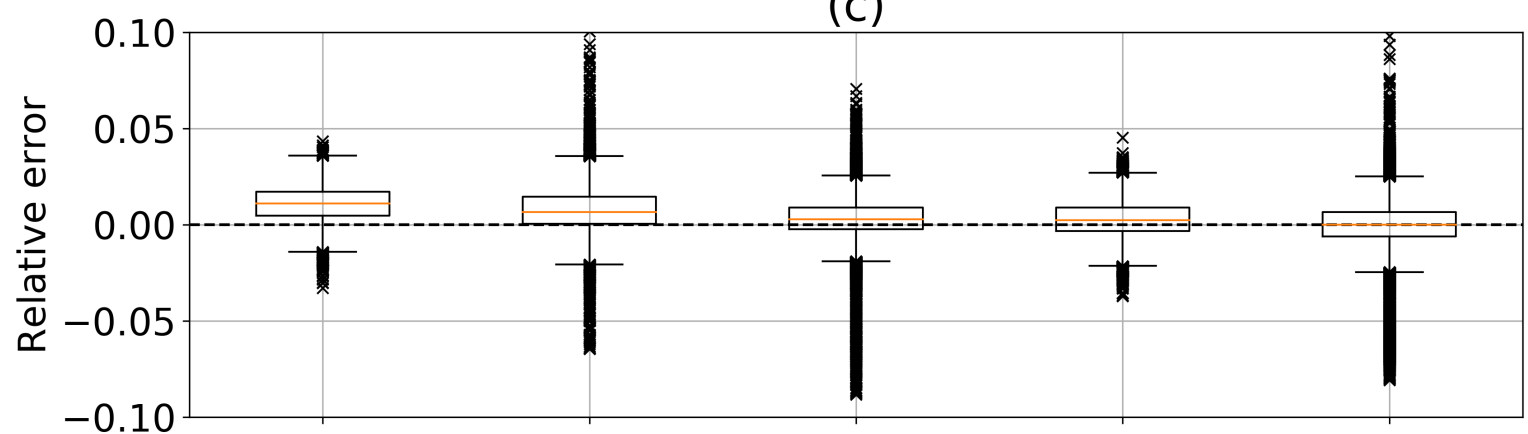

(d)

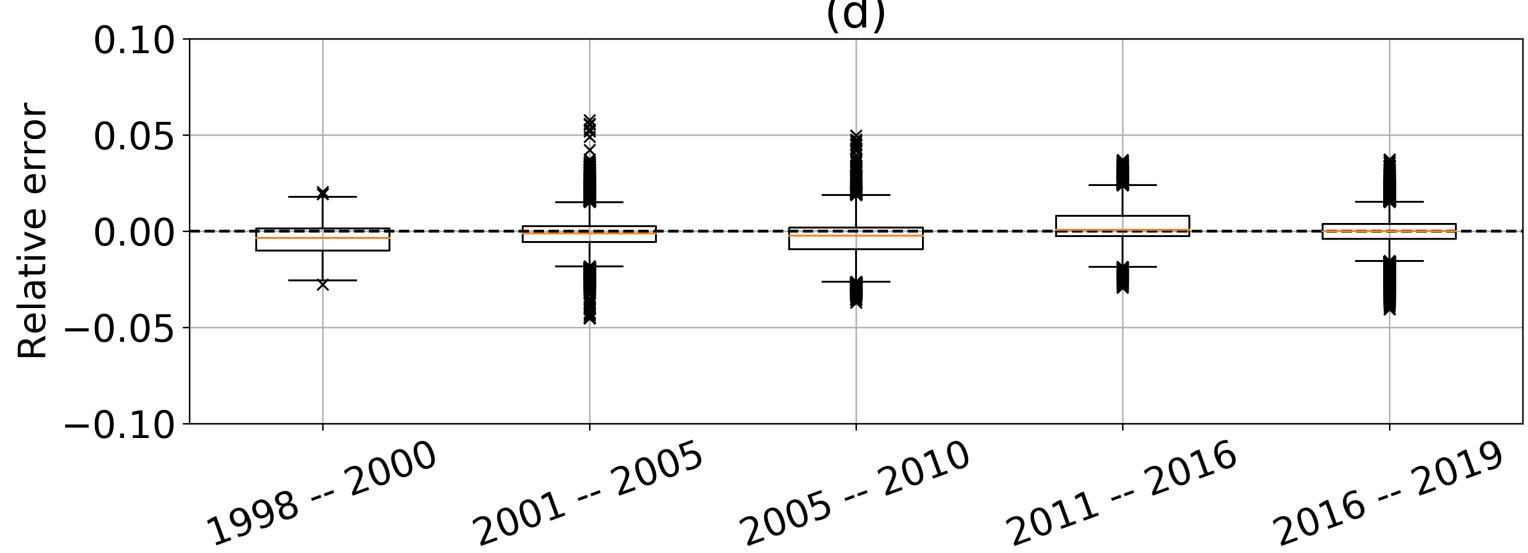

Extended Data Figure 9: Box-and-whiskers plot of relative errors for the predicted DIC concentrations compared to the measured DIC. (a-b) For model trained using GLODAP bottle data only, and (c-d) model trained using both GLODAP bottle data and SOCCOM Argo float data. (a,c) errors between 0-2 km, and (b,d) errors between 2-4 km. In box-plots, center line: median; box limits: upper and lower quartiles; whiskers: $1.5 \times$ interquartile range; crosses: outliers. 
(a)

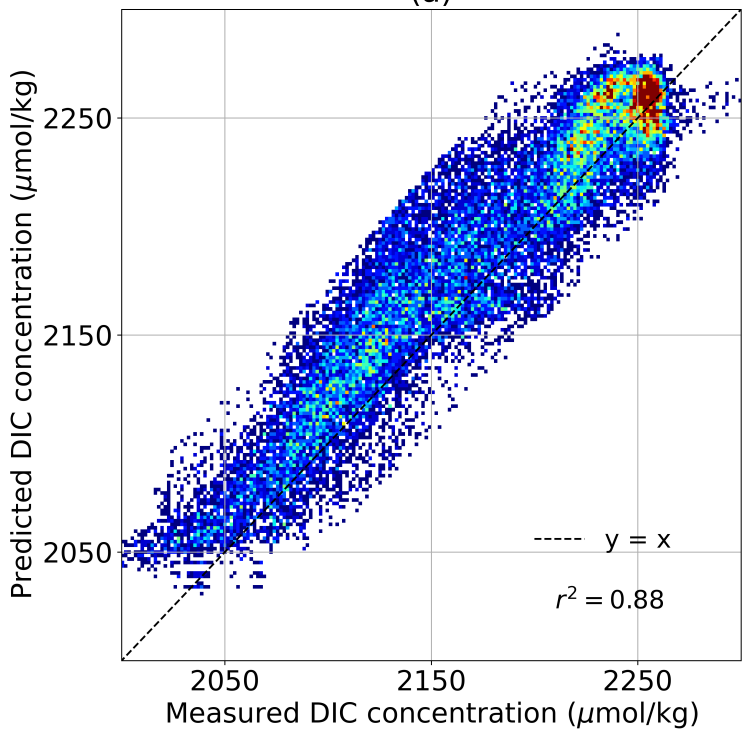

(b)

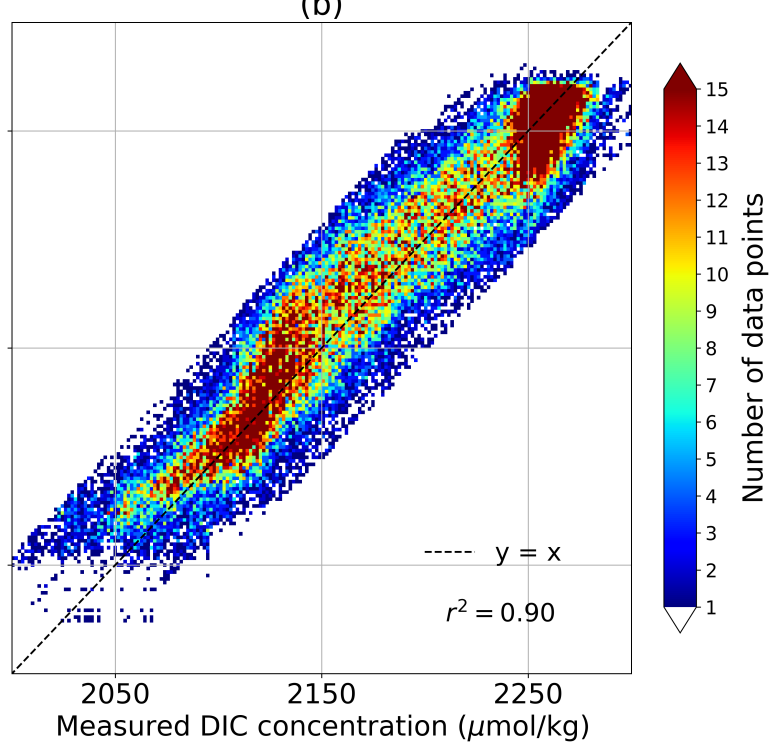

Extended Data Figure 10: Correlation between DIC predicted by the deep learning model and measured DIC. (a) using GLODAP bottle data only, and (b) using bottle data and SOCCOM Argo float data. One-to-one line in plotted in dotted black along with the regression coefficient $r^{2}$. 